\title{
ULTRASTRUCTURE OF THE RETINAL SYNAPSES IN CUBOZOANS
}

\author{
Gregory Clark Gray
}

\author{
A Thesis Submitted to the \\ University of North Carolina Wilmington in Partial Fulfillment \\ of the Requirements for the Degree of \\ Master of Science \\ Department of Biology and Marine Biology \\ University of North Carolina Wilmington \\ 2007
}

Approved by

Advisory Committee

Chair

Accepted by

Dean, Graduate School 
This thesis has been prepared in the style and format consistent with the journal

Biological Bulletin 


\section{TABLE OF CONTENTS}

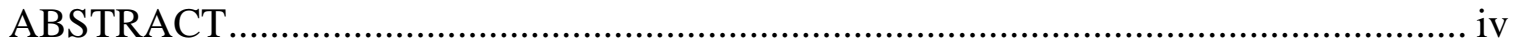

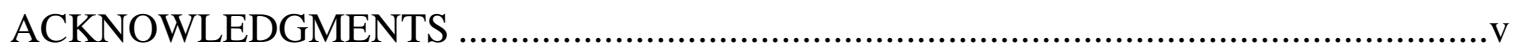

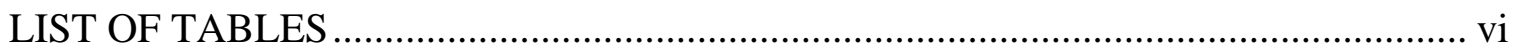

LIST OF FIGURES …….................................................................................... vii

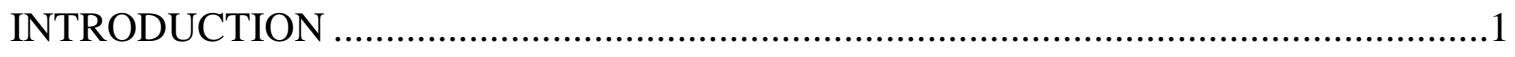

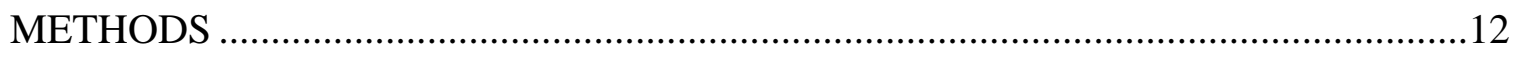

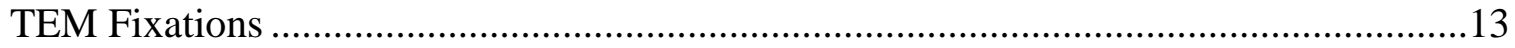

Invaginated Synapse Diameter and Density Analysis .....................................................17

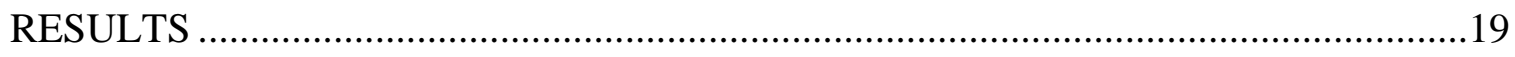

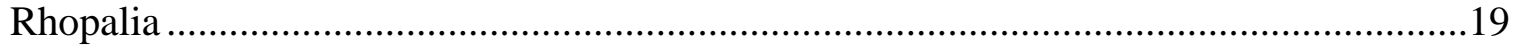

Anatomy of Lensed Eyes ......................................................................................19

Synaptic Morphology in the Upper and Lower Lensed Eye.............................................20

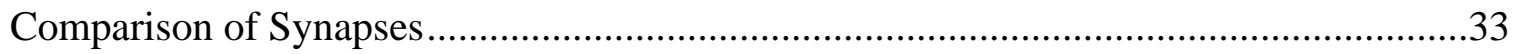

Invaginated Synapse Densities .......................................................................................33

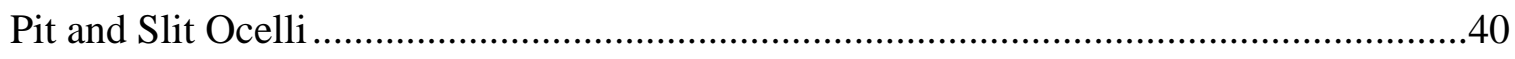

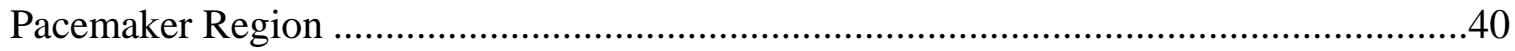

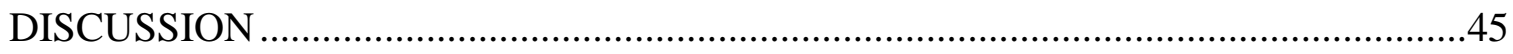

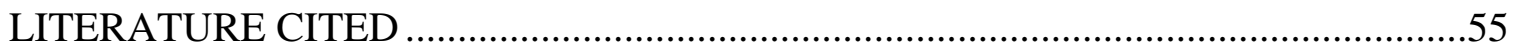




\begin{abstract}
Cubomedusae (box jellyfish) are well known for their strong directional swimming and responses to visual stimuli, and they have complex lensed eyes comparable to those of higher multicellular animals. Cubomedusae possess a total of 24 eyes that are of four morphologically different types, yet little is known about the neural organization of their eyes. The eyes are located on ganglion-like structures called rhopalia - each of the four rhopalia contains an upper and a lower lensed eye (with a cornea, lens and retina), two pit ocelli, and two slit ocelli. Using transmission electron microcopy, the synaptic morphology of the eyes and pacemaker region of four species of cubozoans (Carybdea alata, Carybdea marsupialis, Tripedalia cystophora, and Chiropsalmus quadrumanus) were examined. Similarities in unique invaginated synaptic structures were found in all four species, but only in their upper and lower lensed eyes. Density measurements indicated that the invaginated synapses are located in close proximity to the basal region of photoreceptor cells, and size differences of invaginated synapses were observed between the upper and lower lensed eyes, as well as between species. Four additional types of chemical synapses: clear unidirectional, dense core unidirectional, clear bidirectional, and clear and dense core bidirectional were also observed in the rhopalia of these four species. Results from this study raise the possibility that invaginated synapses may be useful in sorting out the neural circuitry in the upper and lower lensed eyes of these four species.
\end{abstract}




\section{ACKNOWLEDGMENTS}

I want to express my sincere thanks to Dr. Richard Satterlie for taking me on as his graduate student and giving me the opportunity to pursue my graduate degree. Rich, I cannot thank you enough for all the help and guidance you have given me during my time of study under you. You've been an exceptional mentor. I also want to thank my committee members: Dr. Richard Dillaman, for letting me use his lab and allowing me to tap into his wealth of knowledge; Dr. Steve Kinsey, for his guidance in the design of my study; and Dr. Vicki Martin, for sharing her abundance of knowledge about box jellyfish with me. In addition, I am very grateful to Mark Gay for his help in the electron microscopy lab. Without his expertise, I would have never finished this project. To my lab mates: Barbara, Rodney, and Mary - thanks for all your help and support. You guys are great! To all the friends I have made during my years here, thanks for the great memories. To Matilde, thank you for your support throughout this journey. Your positive attitude and smile helped me get through the hardest days. Most of all, I want to thank Mom and Dad. My passion for the beach started with you guys taking me there, and it's where I discovered my first shell. Your continued support and encouragement mean more to me than you will ever

know. I love you! 


\section{LIST OF TABLES}

Table

Page

1. Mean bell height and diameter (cm) from C. alata, C. marsupialis, and

C. quadrumanus...

2. Mean diameter and length measurements of the four types of eyes ......................15

3. Dimensions of the neural layer taken at the mid region

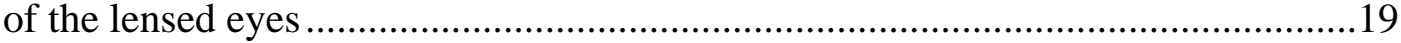

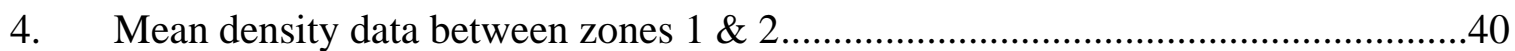

5. Types of synapses found in cubomedusae .......................................................49 


\section{LIST OF FIGURES}

Figure Page

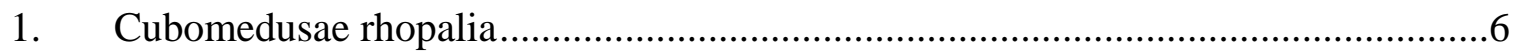

2. Montage of the lower lensed eye of $C$. alata .....................................................

3. Cross section of the lower lensed eye of $C$. marsupialis stained

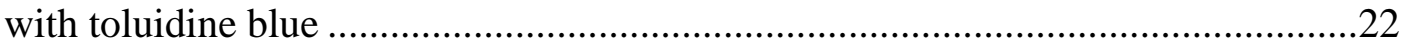

4. Micrographs taken at the same magnification of the upper and lower lensed eye in C. alata ..................................................................25

5. Mid sagittal section of the lower lensed eye of C. alata, showing that both the pre and postsynaptic processes are putative photoreceptors ......................27

6. Sagittal section of the mid region in the lower lensed eye of C. alata, highlighting the basal end of a putative photoreceptor cell invaginating into a nearby cell

7. Invaginated synapse diameters of the upper and lower lensed eye between individual animals

8. Unidirectional synapses found in the neural layer (zone 2) of the upper and lower lensed eye of C. alata

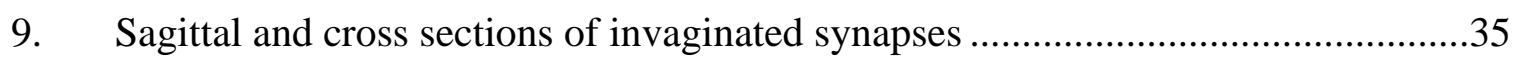

10. Invaginated synapse diameters of the upper and lower lensed eye among species

11. Mean synaptic densities (zones 1 \& 2) of invaginated synapses in the upper and lower lensed eye between individual animals

12. Mean synaptic densities (zones 1 \& 2) of invaginated synapses in the upper and lower lensed eye between species

13. Sagittal sections of C. marsupialis and C. quadrumanus slit ocelli

14. Cross sections of the different types of synapses located in the pit and slit region of C. alata

15. Types of synapses found in the pacemaker region of C. alata 


\section{INTRODUCTION}

The Class Cubozoan (box jellyfish), is within the Phylum, Cnidaria (Werner 1975) along with three other classes, Anthozoa, Hydrozoa, and Scyphozoa. Cubozoans are separated into two families, Carybdeidae and Chirodropidae. This study included three representatives of the family, Carybdeidae (Carybdea alata, Carybdea marsupialis and Tripedalia cystophora), and one member of the family, Chirodropidae (Chiropsalmus quadrumanus).

Cubomedusae are predators, capturing small fish and crustaceans with their potent sting (Larson, 1976). As a group, cubozoans are found in shallow tropical seas throughout the Pacific, Indian, and Atlantic Oceans. They generally occur in quiet, shallow waters, protected bays and estuaries, and over sandy-bottomed shorelines, although some species have been reported in the open ocean (Werner, 1973). Cubomedusae apparently descend to deeper water during daylight hours, but during summer months, adults are often observed at, or near, the surface (Larson, 1976).

Among cnidarians, cubomedusae are unique for their potent sting and strong swimming ability, but it is their eyes and elaborate visual behavior which has received a great deal of recent attention (Larson, 1976; Hamner, 1994; Matsumoto, 1995; Coates, 2003; Garm et al., 2007b). These eyes play a major role in the control of behavioral responses to visual stimuli (Skogh et al., 2006). Cubomedusan behavioral activities, particularly those of feeding, reproduction, and predator avoidance, are affected by light. For example, Tripedalia cystophora are able to navigate around dark objects and stay in the light shafts between mangrove roots (Matsumoto, 1995; Stewart, 1996). When cubomedusae venture into shadows, they reverse course and swim back to the light areas. In laboratory experiments shading them with a hand also causes them to change direction (Martin, 2002). Carybdea and Tripedalia also have been seen moving away 
from dark objects, such as divers and the oars of a boat (Matsumoto, 1995). Chironex fleckeri, has the ability to navigate accurately between pier pilings and take evasive action when people venture too close (Hamner, 1994).

Cubomedusae are capable of directed swimming toward a light source and are reported to be among the fastest and strongest medusan swimmers (Larson, 1976). Barnes (1961) performed laboratory experiments with Chiropsalmus quadrigatus and Chironex fleckeri to examine their turning behavior when exposed to light. Both species oriented to the light of a match, up to $5 \mathrm{ft}$. $(1.52 \mathrm{~m})$ away, even if the match was extinguished before the turning movement was completed. Hamner et al. (1995) found that Chironex fleckeri avoided dark objects positioned in an aquarium, but readily moved toward, and knocked over, white standpipes in the tank. Similar observations have been reported by Garm et al. (2007b). Hamner et al. (1995) found that when black panels were placed at one end of a tank and white panels at the opposite end, the medusae swam away from the black end and toward the white end, whereas if black panels were placed at both ends of a tank, the jellyfish moved to the center of the tank. Photic responses of cubomedusae are believed to play a significant role in feeding behavior since some species are able to orient towards, catch, and eat fish (Larson, 1976).

Cubomedusae have the optical capability to form poor resolution images (Nilsson et al. 2005). Due to the close proximity of the retina to the lens in the lensed eyes, however, it may be impossible for these eyes to form sharp images (Nilsson, 1989). Nilsson et al. (2005) have shown that the ray paths of the lenses in both lensed eyes are severely under-focused and that the focal point falls well beyond the retinal layer. They suggest the lens position is under-focused to remove fine image details so the lens may act as a low-pass filter, allowing the animal to detect large and stationary, or slow moving objects, but not smaller objects such as plankton and small 
particles (Coates and Theobald, 2004; Nilsson et al. 2005). This coincides with Hamner's observations (1994) that Chironex fleckeri swam away from black panels, ranging in sizes from 30,10 , and $1 \mathrm{~cm}$ wide, which were placed in an aquarium. Martin (2002) states that if these lenses are not used to form images, then they may function as light-collecting structures regulating the light-sensitivity threshold of the photoreceptors.

Ultrastructural studies (Nilsson et al. 2005; Parkefelt et al., 2005; Skogh et al., 2006) have shown that cubomedusae have the optical capacity to form images, but it is still unknown where and how the visual information for these behaviors is processed. It has been suggested that the visual information is processed in the subumbrellar nerve ring (Martin, 2002), while behavioral studies suggest that the visual processing takes place in the rhopalia (Hamner et al., 1995; Matsumoto, 1995; Coates, 2004; Garm et al., 2007b). Satterlie (1979) showed that the pacemakers that control swimming movements are located in the rhopalia. Thus, it would seem likely that at least some degree of visual processing would occur in each rhopalium as well. Electrophysiological data suggests that the impulses leaving the rhopalia are much more complex than that of just a pacemaker signal, as other impulses also carry visual information (Garm et al., 2006).

Cubomedusae are radially symmetric animals which have a box-shaped bell. With the exception of Carybdea alata, the swimming bell of Chiropsalmus quadrumanus, Carybdea marsupialis and Tripedalia cystophora is about as tall as it is wide. A four-lobed manubrium, which serves as the mouth, hangs down from the top of the subumbrella. Nonstriated muscular pads, called pedalia, which contain one or more tentacles, are located at the corners of the bell. A circular muscle ring, the velarium, is located along the opening of the bell and is involved in the narrowing of the bell aperture during swimming. Cubozoans are composed of two cellular layers, 
ectoderm and endoderm, which are separated by a layer of connective tissue (mesoglea). The cubomedusan nervous system consists of nerve nets, a subumbrellar nerve ring, and four rhopalia (Conant, 1898; Berger, 1900).

Cubomedusae are not the only jellyfish that have eyes - they are found in both scyphomedusae and hydromedusae as well. These eyes range from simple eyespots, found in scyphomedusae (Matsumoto, 1995; Martin, 2000), to pigment-cups with a lens, found in some hydromedusae (Burr, 1984).

Cubomedusae have four rhopalia, one of which is located on each side of the bell (Fig.1). Each rhopalium contains a balance organ (statolith) and six eyes: two camera - type lensed eyes with cornea, retina, and lens, and four simple eyes (two slit shaped ocelli and two pit shaped ocelli) (Berger, 1898; Conant, 1898). The two camera-type eyes are morphologically very similar to the camera-type eyes found in fish and cephalopods (Martin, 2002; Garm et al., 2007a). All three eye types possess ciliated photoreceptors (Berger, 1898; Yamasu and Yoshida, 1976).

Each rhopalium is attached to the bell by a stalk and resides within a small exumbrellar indentation in the bell, referred to as a niche. All six eyes face inward (towards the center of the bell) but the two lensed eyes face in different directions in the perpendicular plane. The upper lensed eye looks upward, whereas the lower lensed eye looks downward. The two pigment slits are found just above, and on either side of the lower lensed eye, whereas the pigment spots are located on either side of the upper lensed eye. 
Figure 1. Cubomedusan rhopalia. a) The rhopalium is situated in an exumbrellar niche which is attached to the bell by a stalk (arrow). b) A horizontal view depicting the four morphologically different types of eyes: two pit ocelli (p), two slit ocelli (s), upper lensed eye (ule), and lower lensed eye (lle). c) A midline sagittal section of the rhopalium of T. cystophora. The upper lensed eye faces upward through the top of the bell, whereas the lower lensed eye looks downward through the opening of the bell. The pit and slit ocelli cannot be seen. 

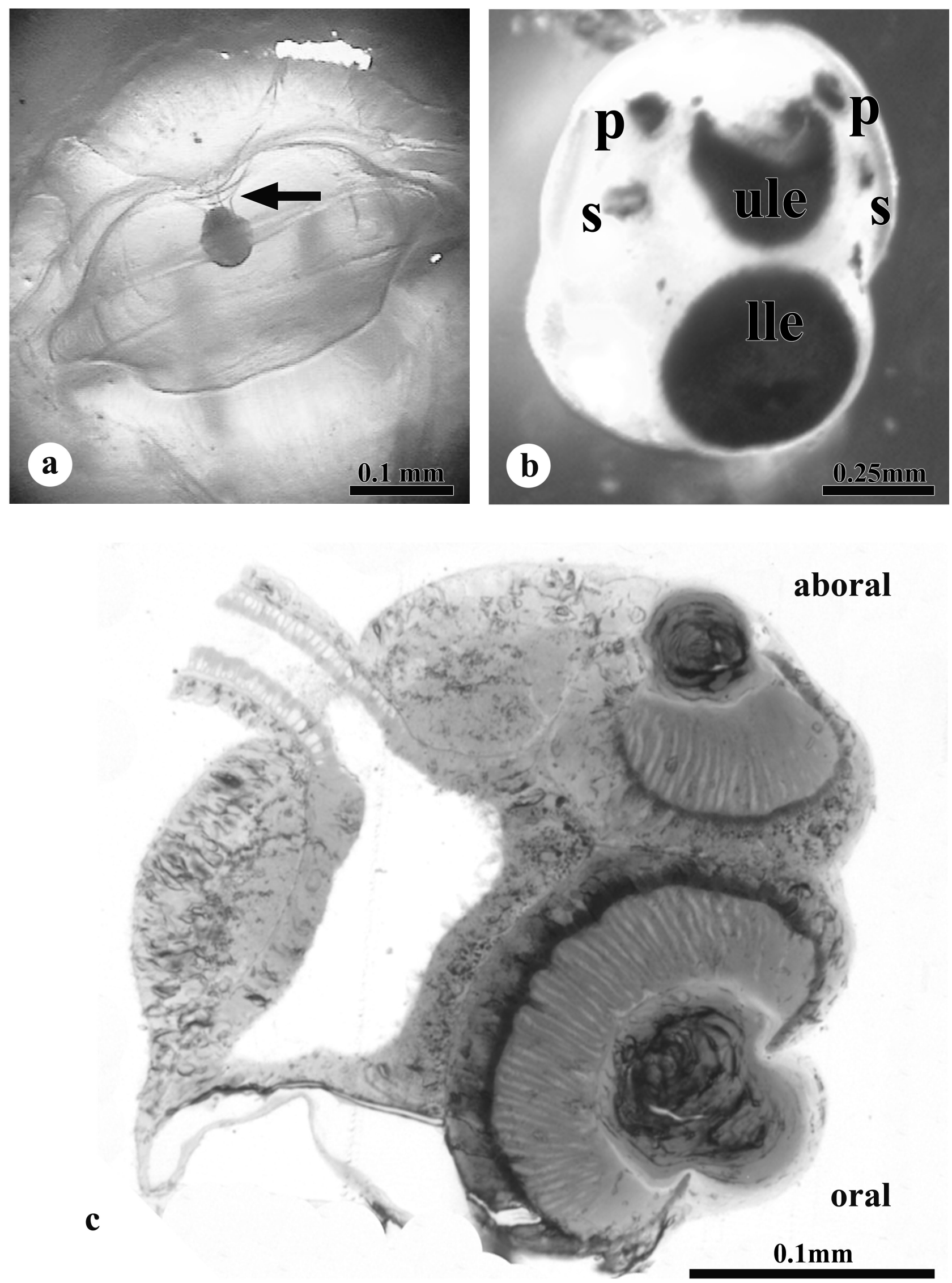
In the lensed eye, the lens is covered by a cornea, which is made up of a layer of simple, squamous epithelial cells bearing numerous microvilli that are continuous with the epithelium of the rhopalium (Berger, 1898; Martin, 2002). The lenses are round, in the center of the eye, flattened at the edges, and composed of crystalline proteins (Piatigorsky et al., 1989; Martin, 2000; Martin, 2002; Nilsson et al, 2005).

In Carybdea marsupialis, the retina is composed of pigmented photoreceptor cells and nonsensory pigment cells (Martin, 2002). All animals appear to share the same photopigment protein, opsin (Land and Nilsson, 2002). Carybdea marsupialis photoreceptor cells are rich in rhodopsin-like and opsin-like photopigments (Martin, 2002). The retinal layer is heterogeneous and consists of three different zones. The receptive zone (zone 1) contains the photoreceptor cells, the synaptic zone (zone 2) contains numerous synapses and mitochondria, and the nonsynaptic zone (zone 3) has neurites with few synapses and mitochondria (Fig.2).

Cubozoan photoreceptors are ciliary with a $9+2$ arrangement of microtubules (Yamasu and Yoshida, 1976) and are made up of a receptive outer segment, a pigmented region, and a nuclear region. The photoreceptors form neurites that enter the underlying neural layer. At least two types of photoreceptor cells are found in the eye, both of which are similar in morphology and contain pigment granules (Martin, 2004).

Due to the extraordinary visual and swimming behaviors of cubozoans, the majority of research has focused on behavioral responses to visual stimuli and physical properties of the lensed eyes, but very little is known about the neural circuitry involved in their visual behavior. What little is known about the neural circuitry of the rhopalium comes from the work of Berger (1898), Yamasu and Yoshida (1976), Parkefelt et al. (2005), Garm et al. (2006), and Skogh et al. (2006). 
Figure 2. a) Montage of the lower lensed eye of $C$. alata depicting the three different zones. b) Enlarged regions of each of the three zones are also shown. A distinct boundary can be seen between zone 2 and 3 due to the sparseness of mitochondria (arrowheads) in zone 3. 

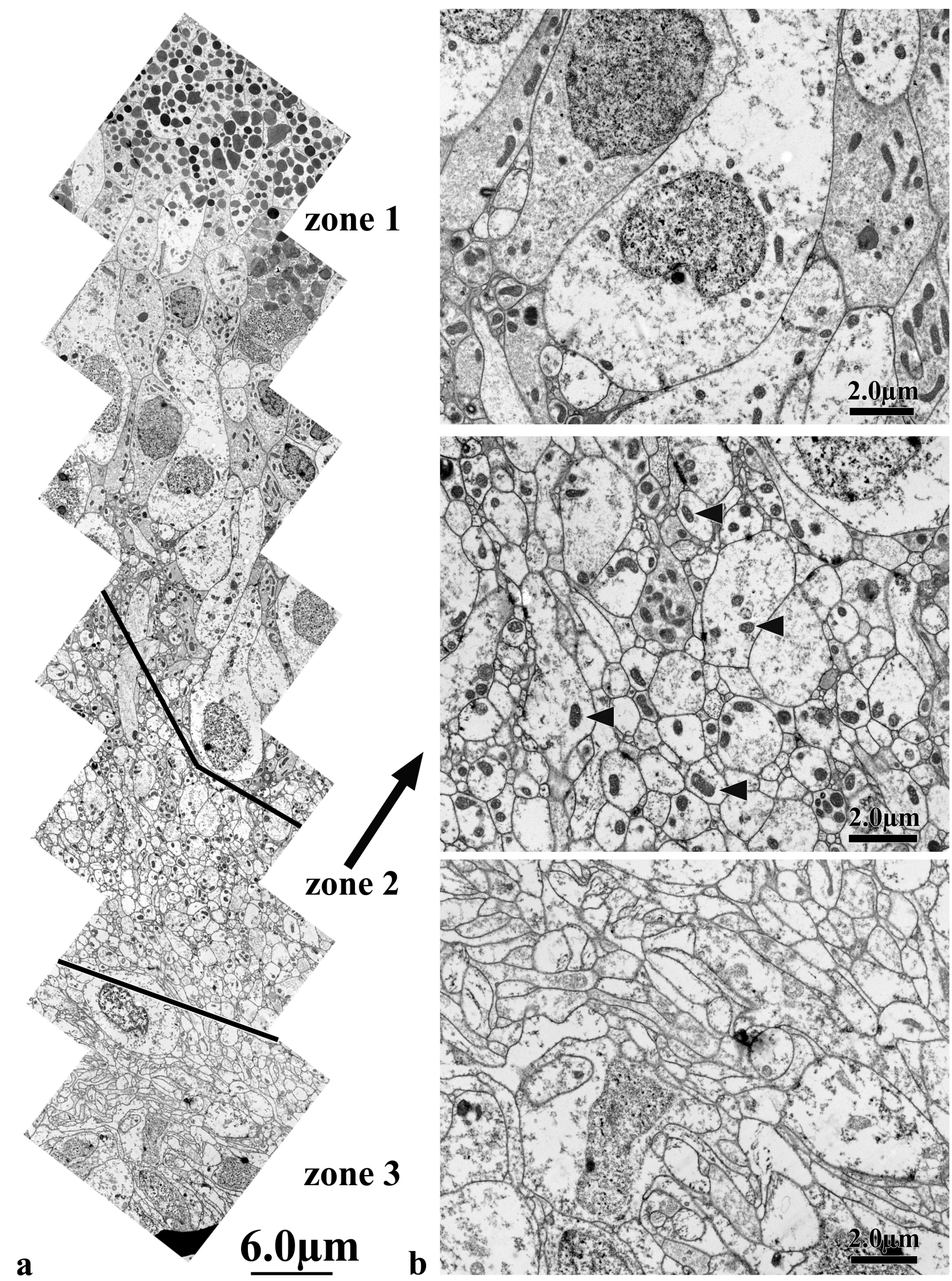

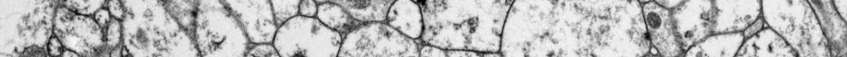

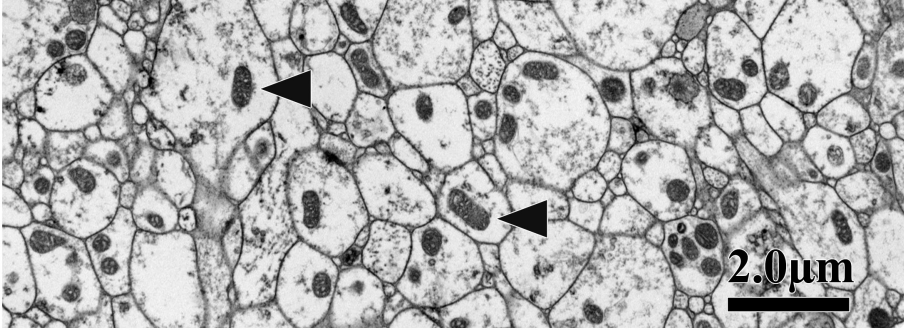

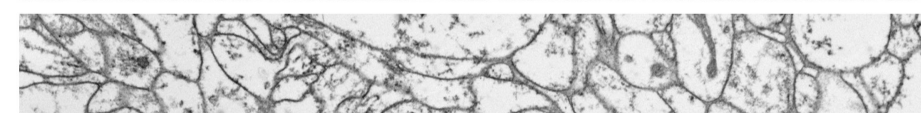

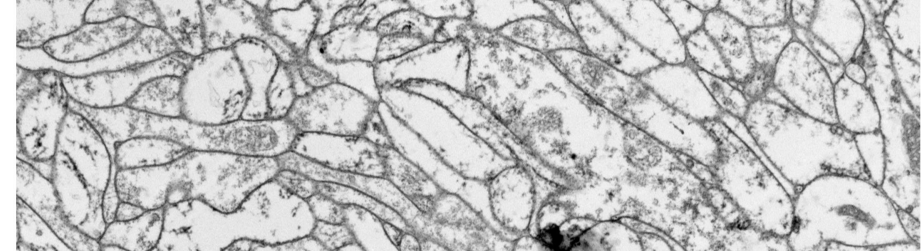

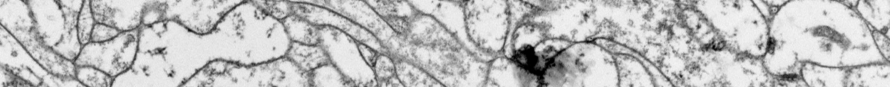

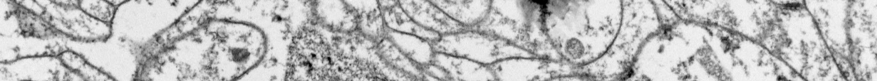

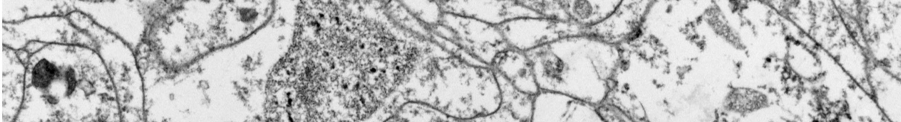

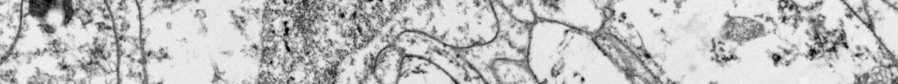

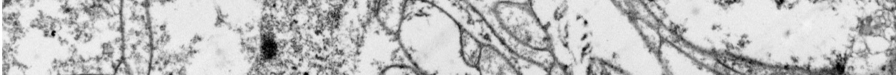

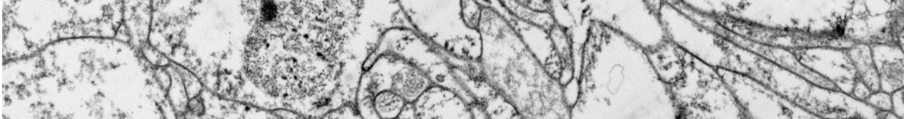

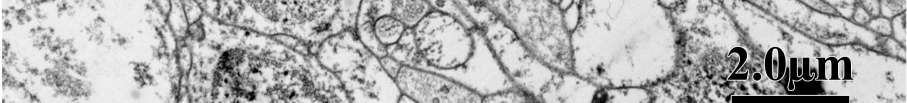

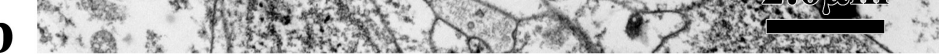


Yamasu and Yoshida (1976) reported that Tamoya bursaria have invaginated synaptic-like junctions between the sensory cells and nerve fibers in the lower lensed eye, but they did not describe the synaptic organization in the other eye structures. Skogh et al. (2006) and Parkefelt et al. (2005) showed that the rhopalium contains a complex nervous system with bilateral neuronal tracts that are closely associated with the pit and slit ocelli, but gave no indication of any synaptic connections with photoreceptor cells. Based on ultrastructure and electrophysiological recordings, Garm et al. (2006) and Parkefelt et al. (2005) indicated that a large part of the CNS is located in the rhopalia and that some of the processing and integration of visual information takes place in the rhopalium.

Although the complex nerve cell populations (Garm et al., 2006; Skogh et al., 2006) and the bilateral neural organization of the rhopalium (Parkefelt et al., 2005) suggest a structural basis for the processing of visual stimuli, more information is needed to fully understand visual processing in cubozoans. Except for a brief description by Yamasu and Yoshida (1976), no other accounts of synaptic organization in cubozoan eyes have been reported.

Invaginated synapses have been observed in several mollusks. These synapses are referred to as spine synapses and consist of a postsynaptic spine invaginating into the presynapstic element, with small clear vesicles aligned along the length of the spine (Bailey and Thompson, 1979; Westfall, 1987). The location of these synapses varies among animals. In squid, the spine synapses are associated with photoreceptors (Cohen, 1973). In Aplysia californica, they are in the siphon nerve and abdominal ganglion (Bailey and Thompson, 1979), and in the octopus, they can be found in both the statocyst and brain (Budelmann and Thies, 1977). The invaginations are also very similar to the ribbon synapses found in many vertebrate photoreceptors, although no presynaptic ribbons are present in cubozoan invaginated synapses. 
The optical system of cubomedusae provides visual information about the world in which they live that is essential for their survival. An important aspect of this information is the ability of the animal to form an image. The clarity of the image does not only depend on the ability of the lens to focus light onto the photoreceptors, but also on the ability of the nervous system to integrate and relay electrical signals from the photoreceptor cells. Thus, it is important to understand the neural organization of the retinal layer to address the question of how visual information is processed.

In cubozoans, vision appears to play an important role in swimming behavior (Hammer et al., 1995; Matsumoto, 1995; Coates, 2004). Visual processing is likely to take place in the rhopalia (Parkefelt et al., 2005). Very little is known, however, of the rhopalial circuitry involved in visual behavior. The complexity of cubomedusan eyes does not correspond with the perception that these animals have relatively simple nervous systems. Recent evidence suggests that the nervous system of cubomedusae may, indeed, be similar to the ganglia of bilateral invertebrates, as directional neural tracts have been described (Parkefelt et al. 2005). This suggests the rhopalial nervous system is much more complex than previously thought.

Yamasu and Yoshida (1976) observed unique invaginated synapses in the lower lensed eye of Tamoya bursaria. A similar observation was made in Carybdea marsupialis (Satterlie, unpublished). It is possible that these unique synapses can be used as markers to help sort out the neural circuitry in the retinal neural layer. Therefore, four species of cubomedusae were examined to test the following hypotheses: (1) are invaginated synapses found only in the lensed eyes of cubomedusae, and (2) are invaginated synapses in close association with photoreceptor cells in the lensed eyes? 
The objective of this study is to examine the synaptic morphology in the rhopalia of cubomedusae to determine if invaginated synapses can be used as markers to help characterize the neural circuitry in the eyes. This study describes the synaptic morphology in the pit, the slit, the lensed eyes, and the pacemaker region of Carybdea alata. Unique invaginated synapses were observed in the upper and lower lensed eyes. Size and distribution of invaginated synapses were taken to provide a more detailed analysis of the synaptic morphology of cubomedusan eyes. Similar data from Chiropsalmus quadrumanus, Carybdea marsupialis, and Tripedalia cystophora was also included for comparison.

\section{METHODS}

Four species of cubomedusae were used: Carybdea alata, Chiropsalmus quadrumanus, Carybdea marsupialis, and Tripedalia cystophora. Carybdea alata and Chiropsalmus quadrumanus were collected between June and November (2005 - 2007) off the coast of North Carolina (Wrightsville Beach, Carolina Beach, and Bald Head Island). Specimens were collected by bottom trawls from a shrimp boat. Tripedalia cystophora, obtained from Puerto Rico, and Carybdea marsupialis, obtained from Santa Barbara, California, were hand collected between July and November (2005 - 2007) and shipped to the University of North Carolina Wilmington for investigation.

The main focus of this study was on Carybdea alata with Chiropsalmus quadrumanus, Carybdea marsupialis, and Tripedalia cystophora used for a comparative analysis of synaptic organization. Five animals from each species were used in this study with the exception of Tripedalia cystophora. No live specimens of $T$. cystophora could be obtained during this study, therefore data was obtained from tissue samples that had been previously fixed in an earlier 
study (2004). Thus, a total of eight rhopalia from $T$. cystophora were examined, but the exact number of individual animals was not known.

Before fixing the animals, the bell heights and diameters of Carybdea alata, Chiropsalmus quadrumanus, and Carybdea marsupialis were measured (Table 1). In each of the following measurements an optical ruler was used. Bell height was measured from the base of the bell opening to the top of the bell, and diameter was measured from the base of the bell near the opening. Also, rhopalial height and eye dimensions were measured (Table 2). The rhopalial height was measured from the base of the stalk to the bottom of the rhopalium. The upper, lower lensed eyes, pit, and slit diameter and/or length were measured by tracing the outer edge of the eye and then filling-in the traced in area using Adobe Photoshop 7.0 software. Diameters and/or lengths of the eyes were measured with Image Pro Plus 6.1 software, which measures every two degrees of the filled-in portion of the eye and calculates the mean diameter and/or length.

\section{TEM Fixations}

Two different protocols were used in the electron microscopy portion of this study because some of the Carybdea marsupialis and Tripedalia cystophora samples had already been fixed from earlier studies. The newer protocol used to fix three of the species (Carybdea alata, Carybdea marsupialis and Chiropsalmus quadrumanus) is discussed first.

The rhopalia were removed and transferred into $2.5 \%$ glutaraldehyde in $0.2 \mathrm{M}$ Millonig’s phosphate buffer (Millonig’s, 1961), pH 7.6 (1000mOsm) overnight, at room temperature. Samples were then washed in 0.2 M Millonig’s phosphate buffer containing 1.0 M sodium chloride, three times, for twenty minutes each. Tissues were post fixed in $1 \%$ osmium tetroxide in $1.25 \%$ sodium bicarbonate buffer, $\mathrm{pH} 7.2$, for one hour at room temperature. Tissue samples were then washed in $1.25 \%$ sodium bicarbonate buffer, $\mathrm{pH} 7.2$, three times, for twenty 
Table 1. Mean bell height and diameter $(\mathrm{cm})$ from three of the species used in this study. No live specimen of $T$. cystophora could be obtained to take measurements. Five animals per species were used (values are mean \pm standard deviation).

\begin{tabular}{lcc}
\hline \multicolumn{1}{l}{ Species } & Height $^{1}$ & Diameter $^{2}$ \\
& & \\
C. alata & $8.25 \pm 1.26$ & $15.87 \pm 3.83$ \\
C. marsupialis & $4.06 \pm 0.34$ & $14.47 \pm 1.13$ \\
C. quadrumanus & $5.20 \pm 0.53$ & $17.01 \pm 2.82$ \\
${ }^{1}$ Bell height was defined as: the base of the bell opening to the top of the bell \\
${ }^{2}$ Diameter was defined as: the base of the bell near the opening.
\end{tabular}


Table 2. Mean diameter and length measurements of the four types of eyes. Five animals from each of the three species were used. No live specimen of T. cystophora could be obtained to take measurements. Values are mean \pm standard deviation

\begin{tabular}{lccc}
\hline \multicolumn{1}{c}{$\begin{array}{c}\text { Species } \\
\text { Number of samples }\end{array}$} & $\begin{array}{c}\text { C. alata } \\
\mathrm{n}=5\end{array}$ & $\begin{array}{c}\text { C. marsupialis } \\
\mathrm{n}=5\end{array}$ & $\begin{array}{c}\text { C. quadrumanus } \\
\mathrm{n}=5\end{array}$ \\
\hline Rhopalia Length (mm) & $0.80 \pm 0.06$ & $0.76 \pm 0.04$ & $0.80 \pm 0.10$ \\
Lower eye Diameter (mm) & $0.41 \pm 0.41$ & $0.40 \pm 0.15$ & $0.35 \pm 0.01$ \\
Upper eye Diameter (mm) & $0.28 \pm 0.11$ & $0.24 \pm 0.08$ & $0.19 \pm 0.03$ \\
Pit Diameter (mm) & $0.07 \pm 0.02$ & $0.08 \pm 0.03$ & $0.06 \pm 0$ \\
Slit Length (mm) & $0.11 \pm 0.02$ & $0.17 \pm 0.07$ & $0.13 \pm 0.01$ \\
\hline
\end{tabular}


minutes each, and then dehydrated in a graded series of ethanol (30\%, $50 \%$, $70 \%$, 80 \%, 95 \%, $100 \%$ ) for thirty minutes each. After dehydrating the tissues, they were placed in a mixture of Spurr epoxy resin (Spurr, 1969) and 100\% ethanol (ratio 1:1) overnight. The tissue was then placed in a mixture of Spurr epoxy resin and 100\% ethanol (ratio 3:1) for six hours. Tissue samples were infiltrated with 100\% Spurr epoxy resin overnight, after which they were embedded in fresh $100 \%$ Spurr epoxy resin and placed in a $70^{\circ} \mathrm{C}$ oven overnight to polymerize.

The Carybdea marsupialis and Tripedalia cystophora protocol involved removal of the rhopalia, which were transferred into 2.5\% glutaraldehyde in $0.2 \mathrm{M}$ Millonig's phosphate buffer (Millonig’s, 1961), pH 7.6 (1000mOsm), for two hours at room temperature. Samples were then washed in 0.2 M Millonig's phosphate buffer containing 0.6 M sodium chloride, three times, for twenty minutes each. Tissues were post fixed in $2 \%$ osmium tetroxide in $2.5 \%$ sodium bicarbonate buffer, $\mathrm{pH}$ 7.2, for one hour at room temperature. After this, tissue samples were washed twice in deionized water for fifteen minutes each and then dehydrated in a graded series of ethanol (30\%, $50 \%, 70 \%, 90 \%, 100 \%)$. After dehydrating the tissues, they were washed twice in propylene oxide for fifteen minutes each and then infiltrated with a mixture of Spurr epoxy resin (Spurr, 1969) and 100\% propylene oxide (ratio 1:1) overnight. The half and half mixture of Spurr epoxy resin and 100\% propylene oxide was removed and replaced with 100\% Spurr epoxy resin. Tissues were left overnight on a rotator, after which they were embedded in fresh $100 \%$ Spurr epoxy resin and placed in a $70^{\circ} \mathrm{C}$ oven overnight to polymerize.

For both fixations, mid-sagittal and transverse, thick sections $(2-4 \mu \mathrm{m})$ of the eyes were cut using a glass knife on an MT2-B microtome. Thin sections (90nm) were then cut with a diamond knife on a Reichert-Jung Ultracut E ultramicrotome and put on formvar coated grids. The sections were post-stained with 2\% uranyl acetate in 50\% ethanol and Reynolds' lead citrate 
(Reynolds, 1963), twenty minutes each, and examined with a Philips CM 12 Transmission Electron Microscope in TEM bright field mode at $80 \mathrm{kV}$. Pictures were taken of synapses of interest and the negatives were developed and digital images were generated by scanning the negatives with a Microtek Scanmaker 8700. Images were processed with Adobe Photoshop 7.0 for digital darkroom processing.

Invaginated Synapses Diameter and Density Analysis

A serial sampling technique (in which alternating thick and thin sections were taken) was used to calculate the diameter and density measurements. Invaginated synapse diameter measurements (postsynaptic element) were taken from synapses that were cut in cross sections from five animals in each of the three species. The postsynaptic element image was traced and then filled-in using Adobe Photoshop 7.0 software to create a binary image. Diameters of invaginated synapses were measured with Image Pro Plus 6.1 software, which measures every two degrees of the filled-in portion of the synapse and calculates the mean diameter. To calculate the number of invaginated synapses per $\mu^{2}$, the mid region of the lower lensed eye was divided into three zones (Fig. 2). The upper lensed eye was only divided into two zones since the neural layer is about half as thick as the lower eye neural layer (Table 3). In both the upper and lower lensed eyes, the first zone consisted of the cell body region of the photoreceptor cells. The final two zones (lower lensed eye only) consisted of the division of the neural layer into the proximal (synaptic zone) region and distal (non synaptic zone) region. The proximal region is defined as the zone closest to the photoreceptor cell bodies, thus the distal region is the zone closest to the gastrovascular cavity. 
Table 3. Dimensions of the neural layer taken at the mid region of the lensed eyes. Mean and standard deviation from five specimens per species.

\begin{tabular}{lccc}
\hline \multicolumn{1}{c}{ Species } & $\begin{array}{c}\text { Number of } \\
\text { samples }\end{array}$ & $\begin{array}{c}\text { Lower lensed eye } \\
\text { Thickness range }(\mu \mathrm{m})\end{array}$ & $\begin{array}{c}\text { Upper lensed eye } \\
\text { Thickness range }(\mu \mathrm{m})\end{array}$ \\
\hline C. alata & 5 & $62.88 \pm 4.17$ & $41.19 \pm 6.18$ \\
C. marsupialis & 5 & $33.85 \pm 3.04$ & $13.69 \pm 1.17$ \\
C. quadrumanus & 5 & $35.80 \pm 10.27$ & $21.75 \pm 6.21$ \\
T. cystophora & 5 & $7.18 \pm 0.38$ & $12.69 \pm 5.70$ \\
\hline
\end{tabular}


Synaptic diameters among individual animals of the same species, as well as among the three different species, were tested for significant differences using two-way analysis of variance (ANOVA). Invaginated synapse densities in the upper and lower lensed eyes and between zones in the lensed eyes of Carybdea alata, Chiropsalmus quadrumanus, and Carybdea marsupialis were also tested for significant differences using two-way (ANOVA). All statistical tests were analyzed with SAS 9.1 software. In all cases, $\mathrm{P}<0.05$ was considered significant. Tripedalia cystophora were not included in the statistical data due to the small number of observations.

\section{RESULTS}

General anatomy of the rhopalium

The rhopalia ranged from 0.73 to $0.98 \mathrm{~mm}$ in diameter and were attached to the bell by a stalk ranging from 0.28 to $0.42 \mathrm{~mm}$ in length. In all four species each rhopalium resides in a small exumbrellar niche and contains six eyes of four morphologically different types (two pit, two slits, an upper lensed eye, and a lower lensed eye).Two of the eye types, the upper and lower lensed eyes, are camera type eyes with a cornea, retina, and lens. The pit and slit ocelli contain photoreceptor cells along with nonsensory cells.

General anatomy of the lensed eyes

The diameters of the lower lensed eyes, in Carybdea alata ranged from 300-600 $\mu$ m, whereas the upper lensed eyes had diameters of 200-400 $\mu \mathrm{m}$. Variation in eye size exists between cubomedusae species, but the lower lensed eye was almost always a third larger than the upper lensed eye (Table 2). The upper and lower lensed eyes of cubozoans consisted of a cornea, 
spherical lens, retinal layer (consisting of ciliated photoreceptors cells), and a neural layer (Fig. 3). The retina was divided into the following three regions: the vitreous layer, which contains a modified cilium with a $9+2$ arrangement of microtubules and vacuoles; a pigment layer that was filled with pigment granules, tubular mitochondria, Golgi apparatus, and microtubules; and the basal end which branched into the neural layer.

Synaptic morphology in the upper and lower lensed eye

Synapses are easily identified at the electron microscopic level by the presence of spherical or oval shaped synaptic vesicles, a synaptic cleft, and the presence of electron- dense, thickened membranes in an otherwise relatively clear cytoplasm. When the lower and upper lensed eyes of Carybdea alata were cross and sagittally sectioned, finger-like invaginated synapses were found in the neural layer (Fig. 4). The presence of synaptic vesicles and synaptic clefts suggested that these synapses use a chemical mode of transmission. The postsynaptic terminals included fine tubular endings which had diameters of 0.08-0.23 $\mu$ m (mean $=0.154$, S.D. $=0.005, \mathrm{~N}=243$ ) in the lower lensed eye and $0.09-0.27 \mu \mathrm{m}$ (mean $=0.170$, S.D. $=0.001, \mathrm{~N}=68)$ in the upper lensed eye. The longest invagination into a presynaptic photoreceptor cell extended to a depth of $0.629 \mu \mathrm{m}$ (Fig. 5). Clear vesicles, with diameters of $40-70 \mathrm{~nm}$, were found in the presynaptic terminals surrounding the entire length of the finger-like invaginations. The synaptic cleft had a width of 10-17 nm and contained intracleft transverse filaments. In the synaptic region, membranes had a dense, grainy appearance, which extended along the entire length of the invagination on both the pre and postsynaptic membranes. Mitochondria with tubular cristae were often present near these synaptic terminals. Unfortunately, in the majority of the cases, cell types representing the pre and postsynaptic terminals could not be identified. 
Figure 3. Cross section of the lower lensed eye of $C$ marsupialis stained with toluidine blue. The lens (l), retina (r) including pigment layer (pl), and neural layer (nl) are shown.

The separation between the retina and lens is an artifact. 


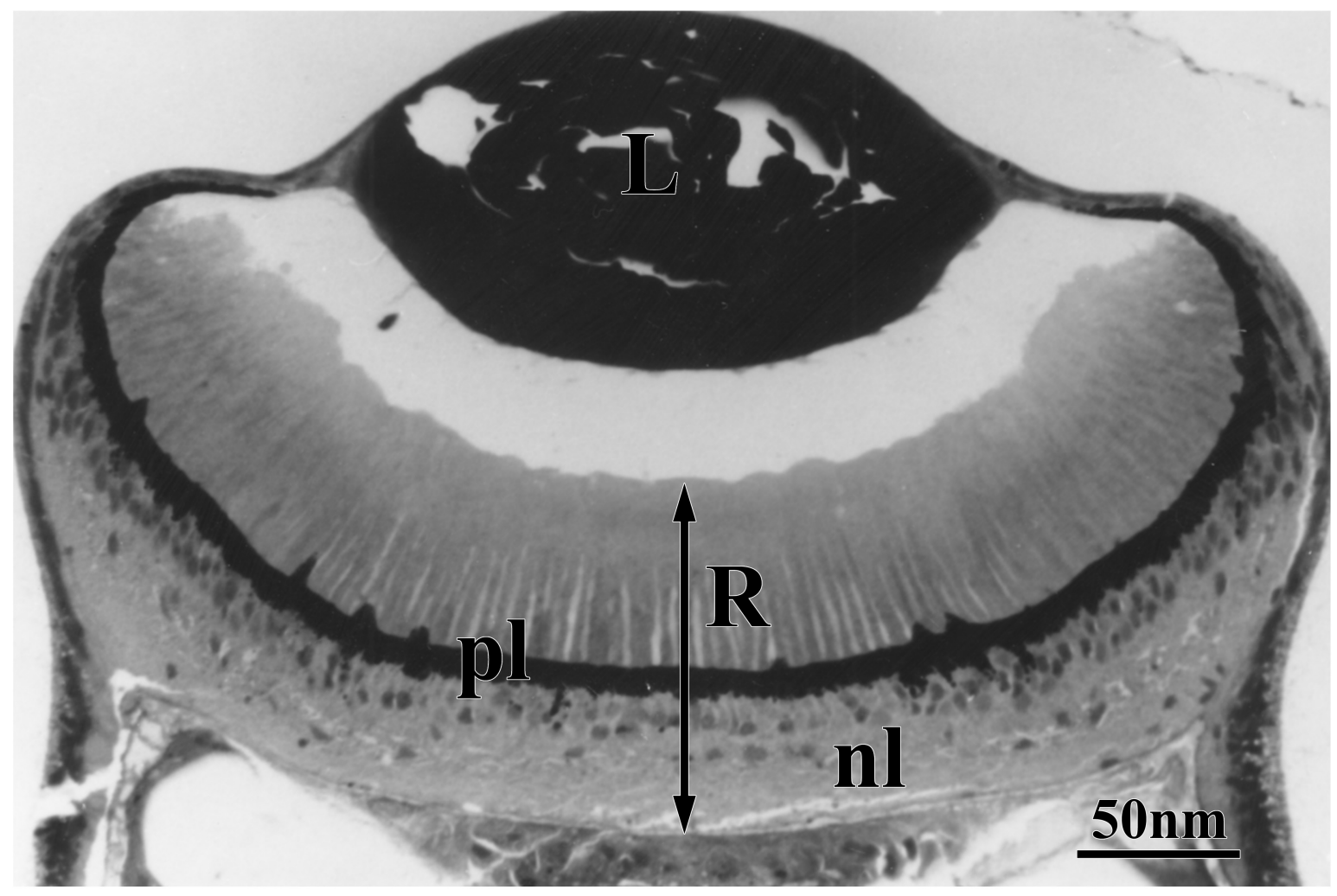


Figure 4. Micrographs taken at the same magnification of the upper a) and lower b) lensed eye in C. alata. Both micrographs are from the mid region of the neural layer. Note the difference in the number of invaginated synapses (arrowheads) present (lower lensed eye $=7$; upper lensed eye $=1)$. 

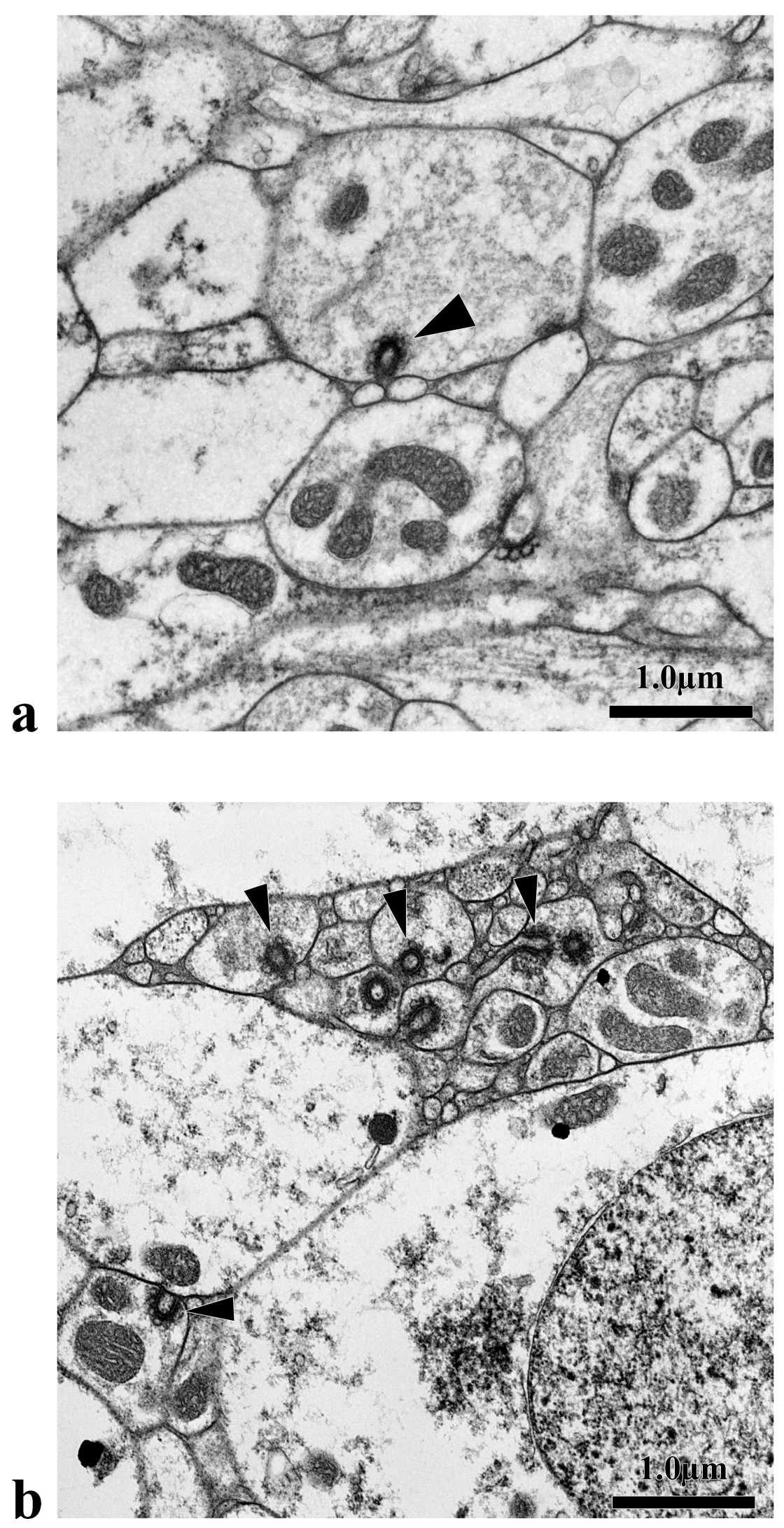
Figure 5. Mid-sagittal section of the lower lensed eye of C. alata. a) Both the pre and postsynaptic processes are putative photoreceptors cells (pc). b) Postsynaptic cell invaginates into the presynaptic cell to a depth of $0.629 \mu \mathrm{m}$. Clear vesicles are present along the length of the synapse. A slight narrowing occurs in the width at the base of the postsynaptic process (arrow). 

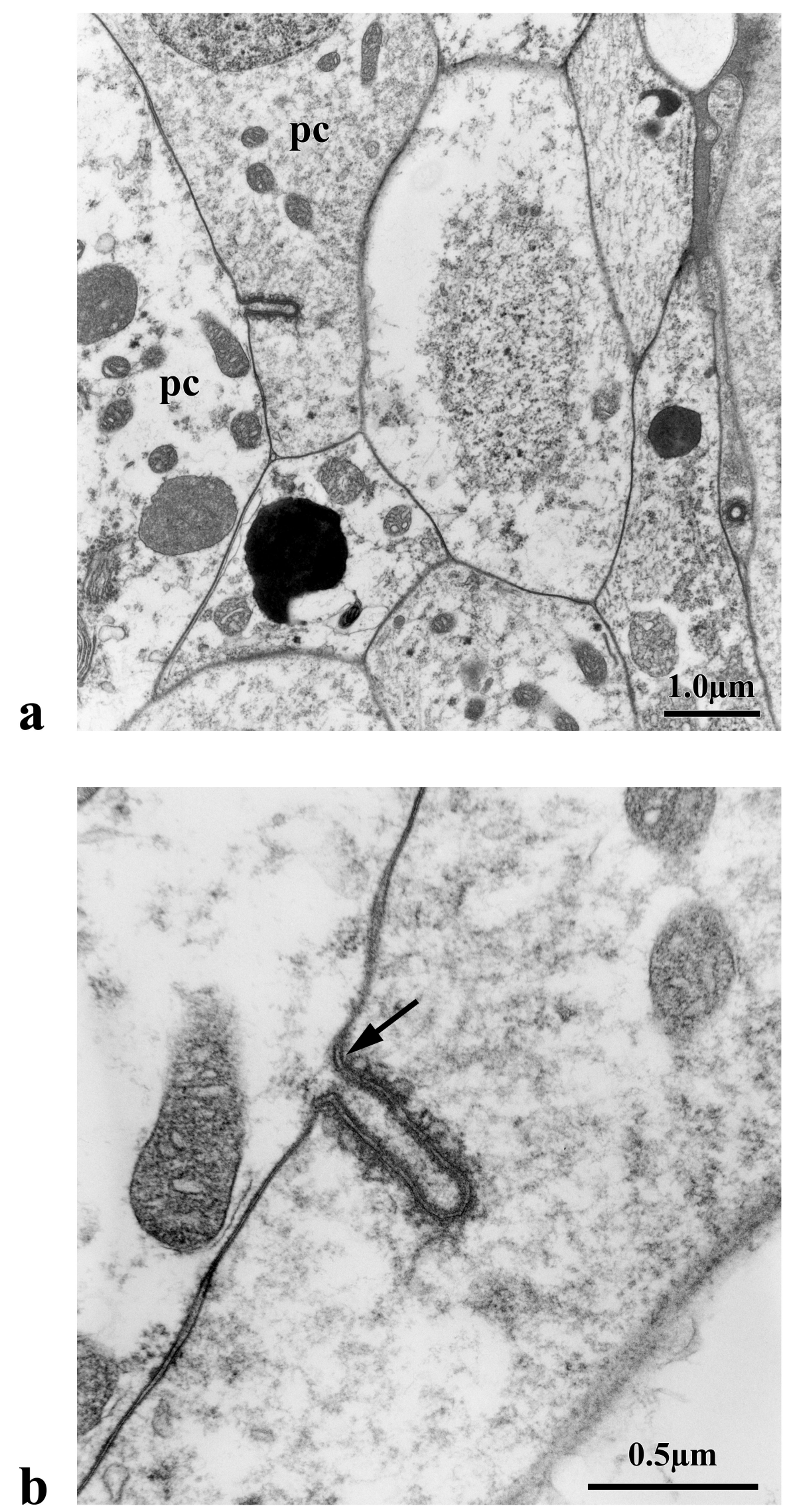
Putative photoreceptor cells, however, were identified as the pre and postsynaptic elements in both the upper and lower lensed eyes of some preparations (Fig. 5). On more than one occasion, photoreceptor cells were seen sending out finger-like processes into neighboring cells (Fig. 6).

Significant differences in invaginated synapse diameters were seen between the upper and lower lensed eyes in Carybdea alata (Fig. 7), with smaller invaginated synapses observed in the lower lensed eye.

In most cases only one invaginated synapse was observed per cell process, but in some cases two or three invaginated synapses occurred together within the same process (Fig. 8). In both the upper and lower lensed eyes, invaginated synapses were located along the sides and mid region of the eyes. Invaginated synapse frequencies decreased and were not observed in the upper portions (near the lens) in the lensed eyes. This is also where fewer photoreceptor cells were found.

In addition to the finger-like invaginated synapses, a second type of synapse was observed in the neural layer of both the upper and lower lensed eyes (Fig. 8). These synapses contained both clear and dense core vesicles, which were found only on the presynaptic cell. The vesicles formed a single layer along the presynaptic membrane and were surrounded by a granular, dense matrix that characterizes the synaptic membrane. The synaptic cleft contained intracleft transverse filaments and vesicle sizes (upper- $0.05 \mu \mathrm{m}$, lower-0.06 $\mu \mathrm{m}$ ) were similar in diameter to those of the invaginated synapses. 
Figure 6. Sagittal section of the mid region in the lower lensed eye of C. alata. a) Basal end of a putative photoreceptor cell (pc). b) Postsynaptic process (arrow) at the basal end of a putative photoreceptor cell invaginating into a nearby cell. Presynaptic cell could not be identified, but does have mitochondria (m) with tubular cristae which are the same as those found in photoreceptor cells. Arrowheads show invaginated synapses in the surrounding cells. 

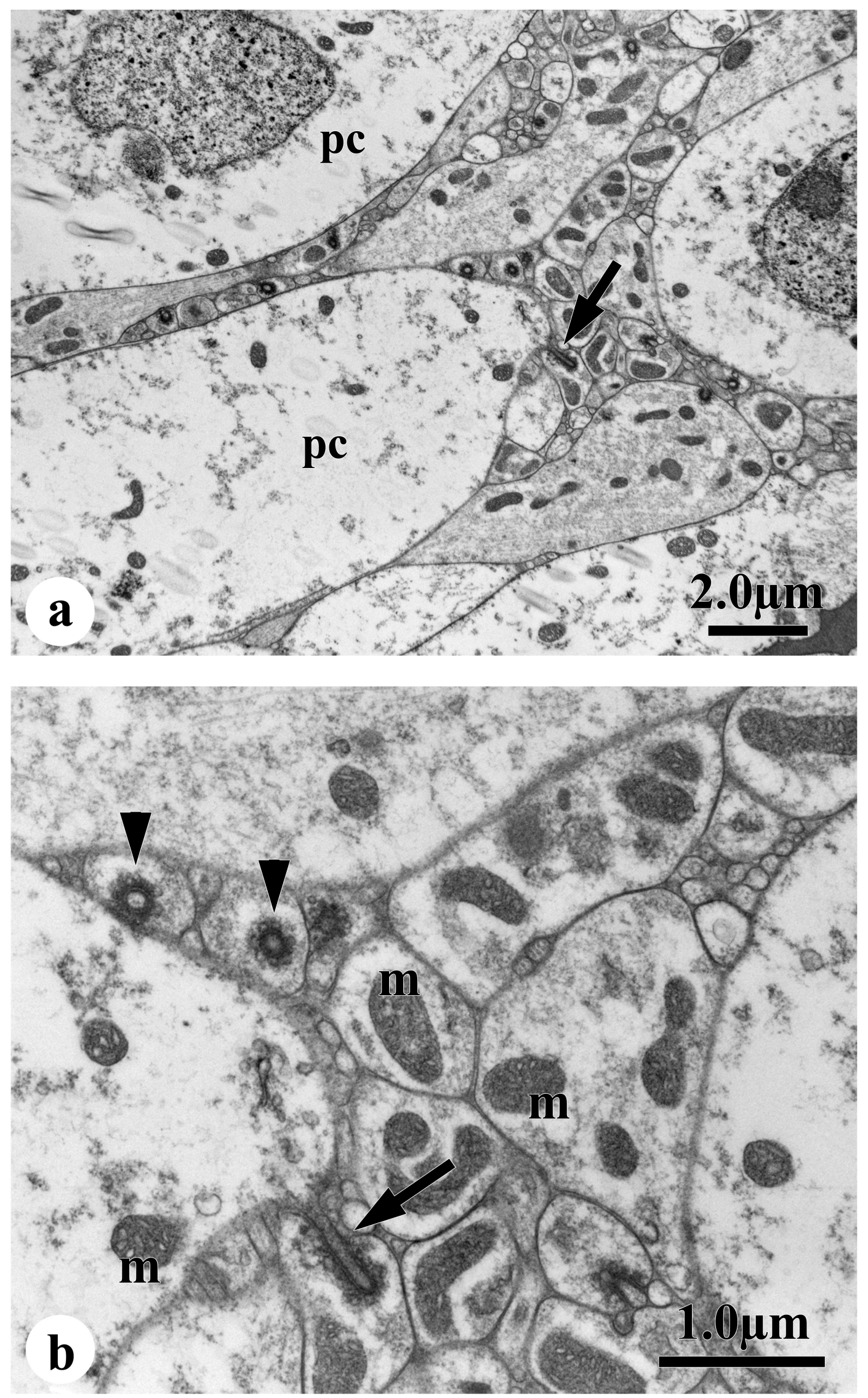


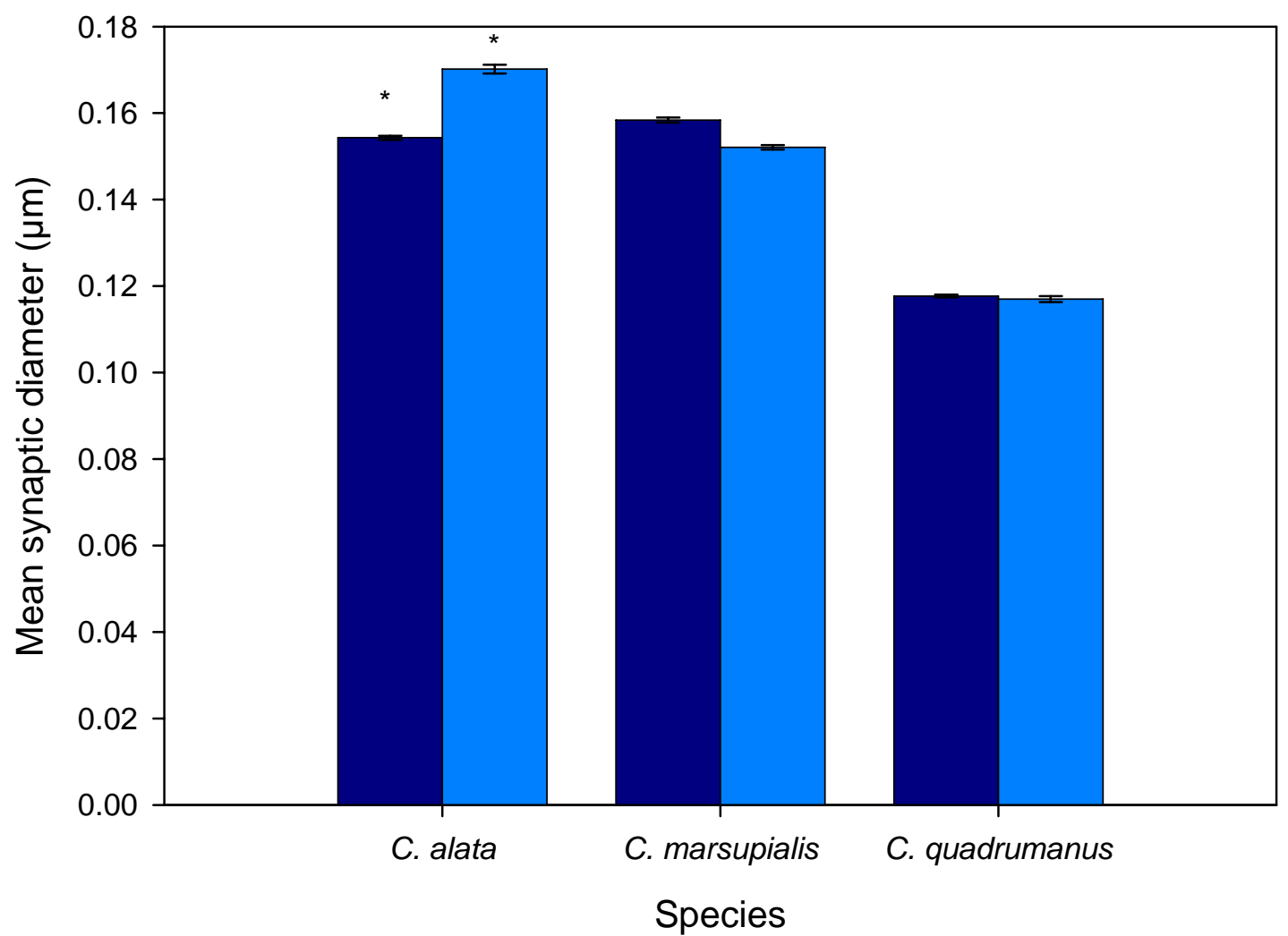

Lower lensed eye Upper lensed eye

Figure 7. Mean diameters of invaginated synapses in upper and lower lensed eyes. Asterisk indicates significant differences $(\mathrm{p}<0.05)$ between eyes in the same species. 
Figure 8. Unidirectional synapses found in the neural layer (zone 2) of the upper (a) and lower (b) lensed eye of C. alata. a) Dense core vesicles (arrowheads) form a single layer along the presynaptic membrane. b) (arrow) Unidirectional synapse containing clear vesicles in the lower lensed eye. Also, multiple postsynaptic cells can be seen invaginating a single presynaptic cell in the lower lensed eye (arrowheads). 

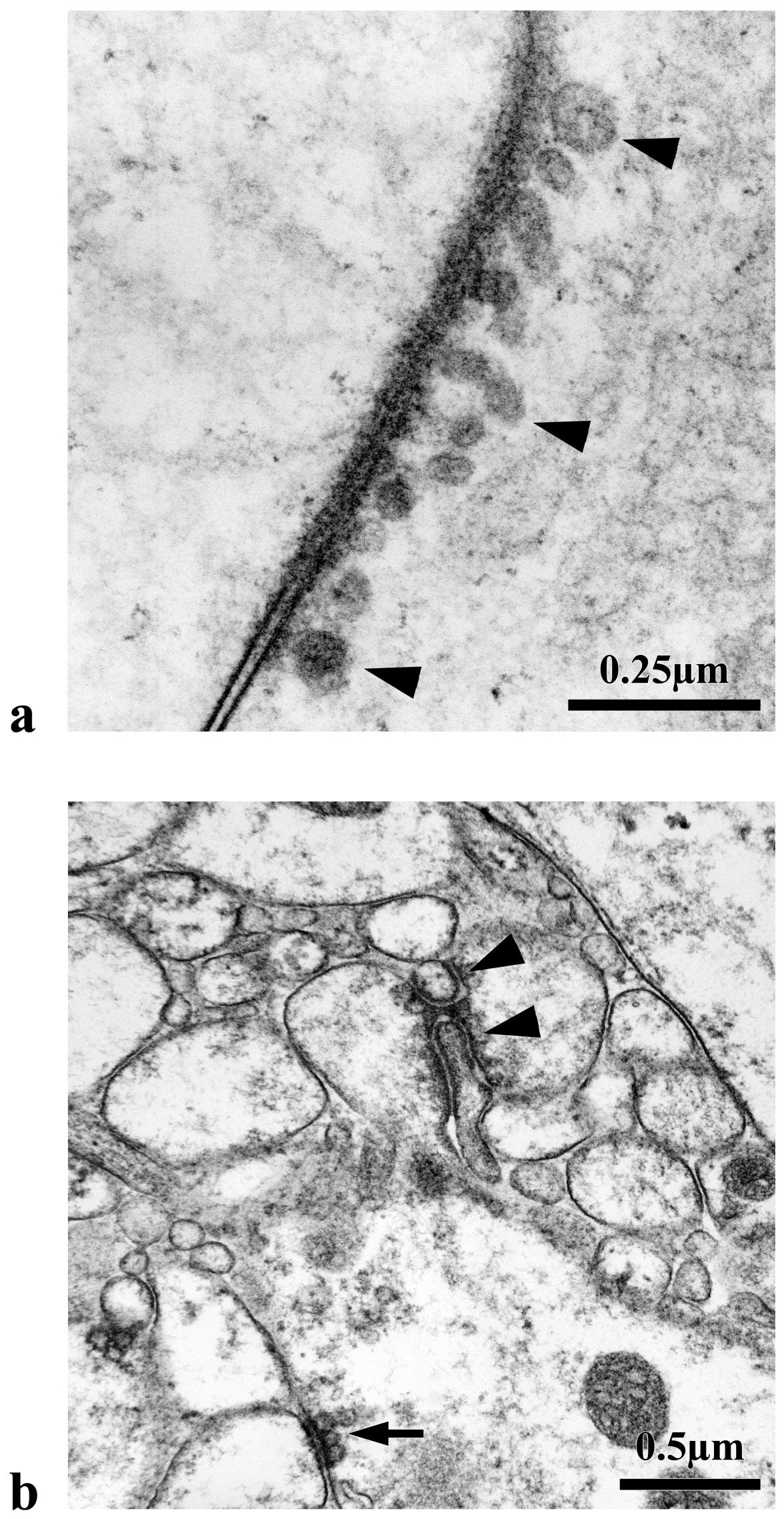


\section{Comparison of synapses}

Invaginated synapses, similar in morphology to Carybdea alata, were also found in the lower and upper lensed eyes of Chiropsalmus quadrumanus, Carybdea marsupialis, and Tripedalia cystophora (Fig. 9). Unlike Carybdea alata, (in which the upper lensed eye had larger diameter invaginated synapses) the diameters of the invaginated synapses were not significantly different between the upper and lower lensed eyes in Chiropsalmus quadrumanus and Carybdea marsupialis (Fig. 7). In comparing invaginated synapse sizes among species, however, the synapses observed in Chiropsalmus quadrumanus were smaller than those found in Carybdea alata and Carybdea marsupialis (Fig.10).

Invaginated synapse densities

When the retinal layers of Carybdea alata, Carybdea marsupialis, and Chiropsalmus quadrumanus were divided into zones (see Methods), variations in invaginated synapse distribution were seen between eyes (Fig. 11), between species (Fig. 12) and between zones (Table 4). In both Carybdea alata and Chiropsalmus quadrumanus, the density of the invaginated synapses was about 50\% greater in the lower lensed eye. This, however, was not the case in Carybdea marsupialis, where no significant differences were seen between the lensed eyes. In comparing densities between zones, in all four species, no invaginated synapses were observed in zone 3. Higher densities of invaginated synapses were seen, however, in zone 2 in all but two cases (Carybdea marsupialis and Carybdea alata in the upper lensed eyes). Significant differences between zone 1 and 2 were observed only in the lower lensed eye of Carybdea marsupialis and Chiropsalmus quadrumanus (Table 4). 
Figure 9. Sagittal (a, b) and cross sections (c) of invaginated synapses: a) C. quadrumanus, b) $C$. alata, and c) C. marsupialis. All three species have similar morphology: membrane thickening, clear vesicles (arrowheads) located along the presynaptic membrane, and intra cleft filaments. 

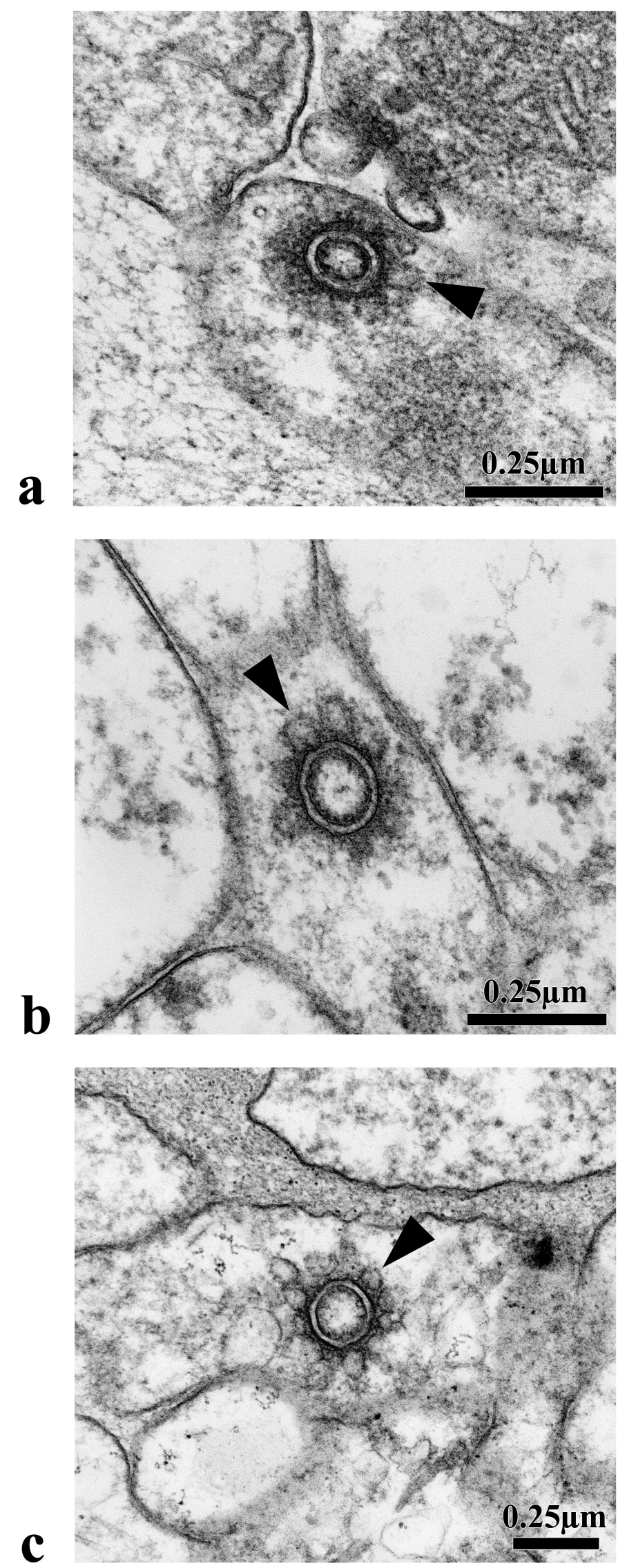


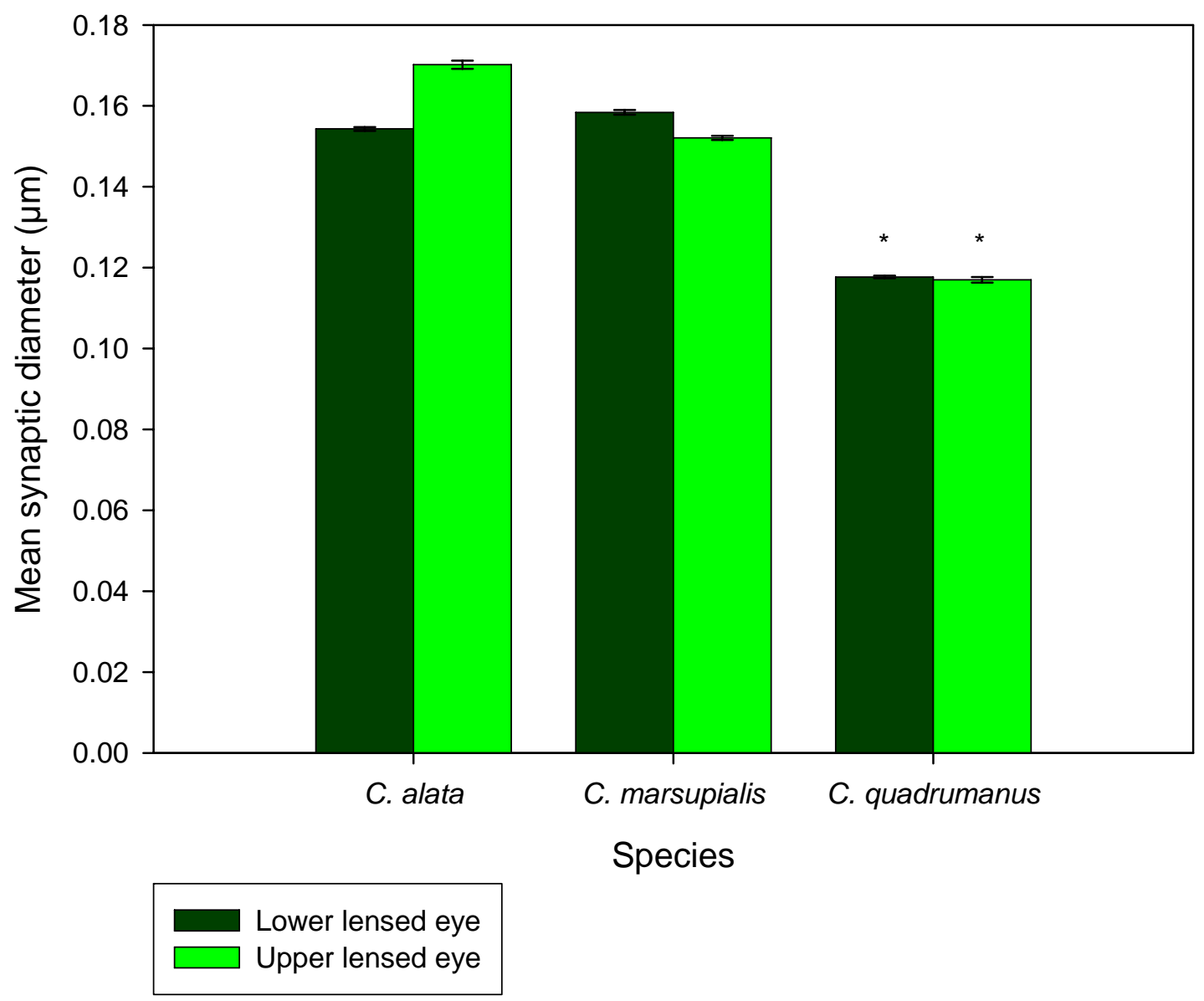

Figure 10. Mean diameters of invaginated synapses in the upper and lower lensed eyes. Asterisk indicates significant differences $(\mathrm{p}<0.05)$ between species. 


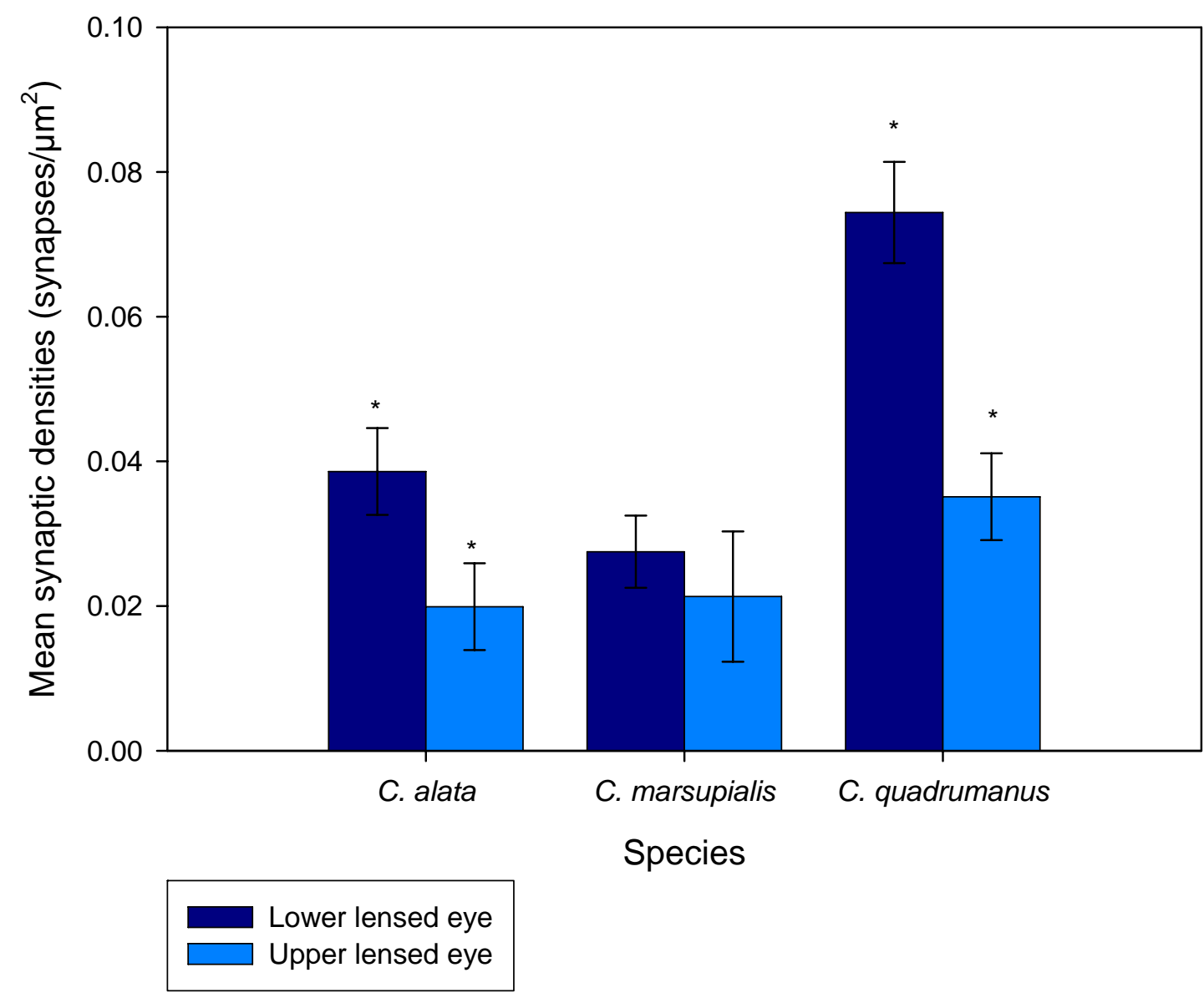

Figure 11. Mean synaptic densities (include both zones 1 \& 2) of invaginated synapses in the upper and lower lensed eyes among individual animals. Asterisk indicates significant differences $(\mathrm{p}<0.05)$ between eyes. 


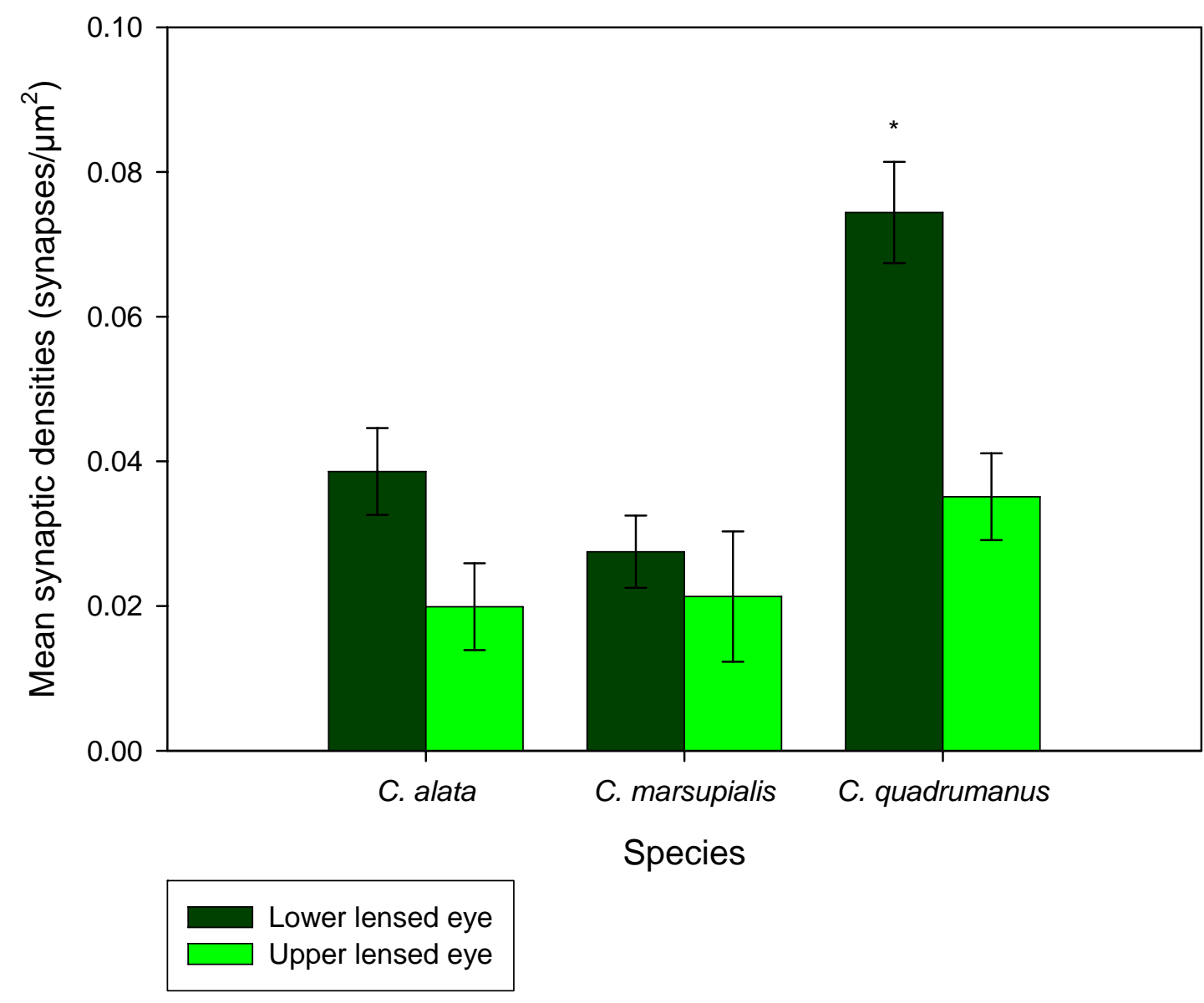

Figure 12. Mean synaptic densities (include both zones 1 \& 2) of invaginated synapses in the upper and lower lensed eyes among species. Asterisk indicates significant differences $(\mathrm{p}<0.05)$ among species. 
Table 4. Mean density data between zones $1 \& 2$. Asterisk indicates significant differences $(p<0.05)$ between zones.

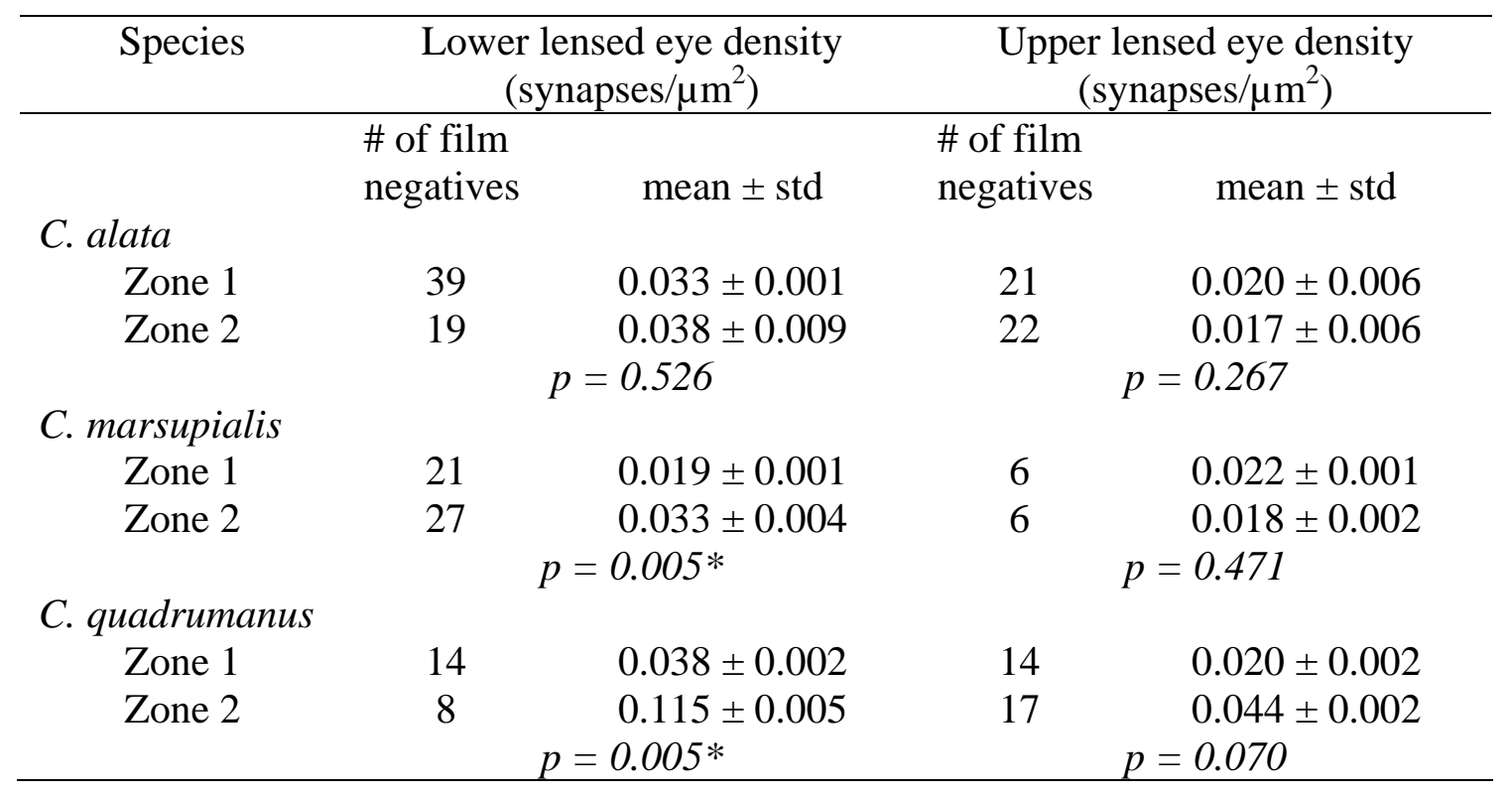


Fewer mitochondria were observed in zone 3. This is not the case in zone 1 and 2, however, in which mitochondria are commonly found along with the majority of invaginated synapses (Fig. 2).

Pit and slit ocelli

Morphological differences in the size of the slit and pit ocelli were seen among species. In Carybdea marsupialis and Tripedalia cystophora, the slits have a rudimentary lens, which is not present in the other two species (Fig. 13). Ciliated epithelial cells, similar in morphology to those observed in the lensed eyes, lined both the slit and pit ocelli. In all four species no invaginated synapses were seen in either the pit or slit ocelli. Basal processes of the photoreceptors projected into a neural layer located directly below the photoreceptor cells. Due to the asymmetrical distribution of synaptic vesicles, this layer consisted primarily of polarized synapses, although bidirectional synapses were also found. Dense and clear core vesicles, ranging from $0.04 \mu \mathrm{m}-$ $0.09 \mu \mathrm{m}$ in diameter, were found in the cytoplasm on the presynaptic cells. Figure 14 shows the various types of synapses that were found in this region. The vesicles were arranged either in a single row, or in a tiered configuration, along the presynaptic membrane. Both the pre and postsynaptic membranes showed a thickening at the synaptic junction. The synaptic cleft contained dense bars or particles that ran across the cleft.

\section{Pacemaker region}

In cubozoans, the pacemaker region is located in the upper region of the rhopalia, near the emergence of the rhopalial stalk (Satterlie, 1979). It consists primarily of an outer layer of nerve cell bodies and a tangle of neurites that are referred to as the rhopalial neuropil. 
Figure 13. Sagittal sections of the slit ocelli a) C. marsupialis and b) C. quadrumanus. A rudimentary lens is present only in C. marsupialis (arrow). Abbreviations (pl) pigment layer, (pr) photoreceptor cell bodies, and (nl) neural layer. 

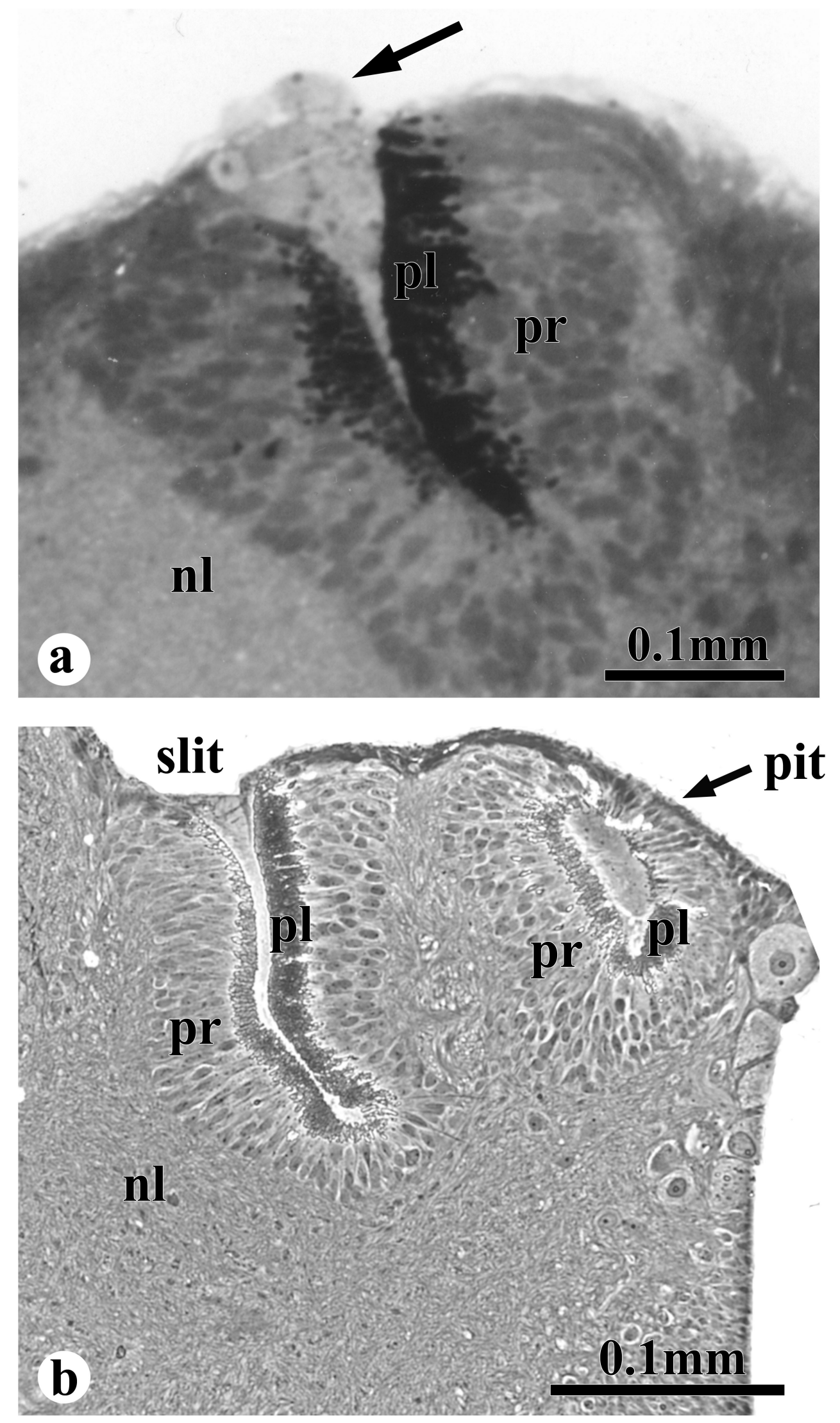
Figure 14. Cross sections of the different types of synapses located in the pit and slit region of $C$. alata. Both unidirectional and bidirectional (arrowhead) synapses are present. (a \& b) pit region, (c \& d) slit region 

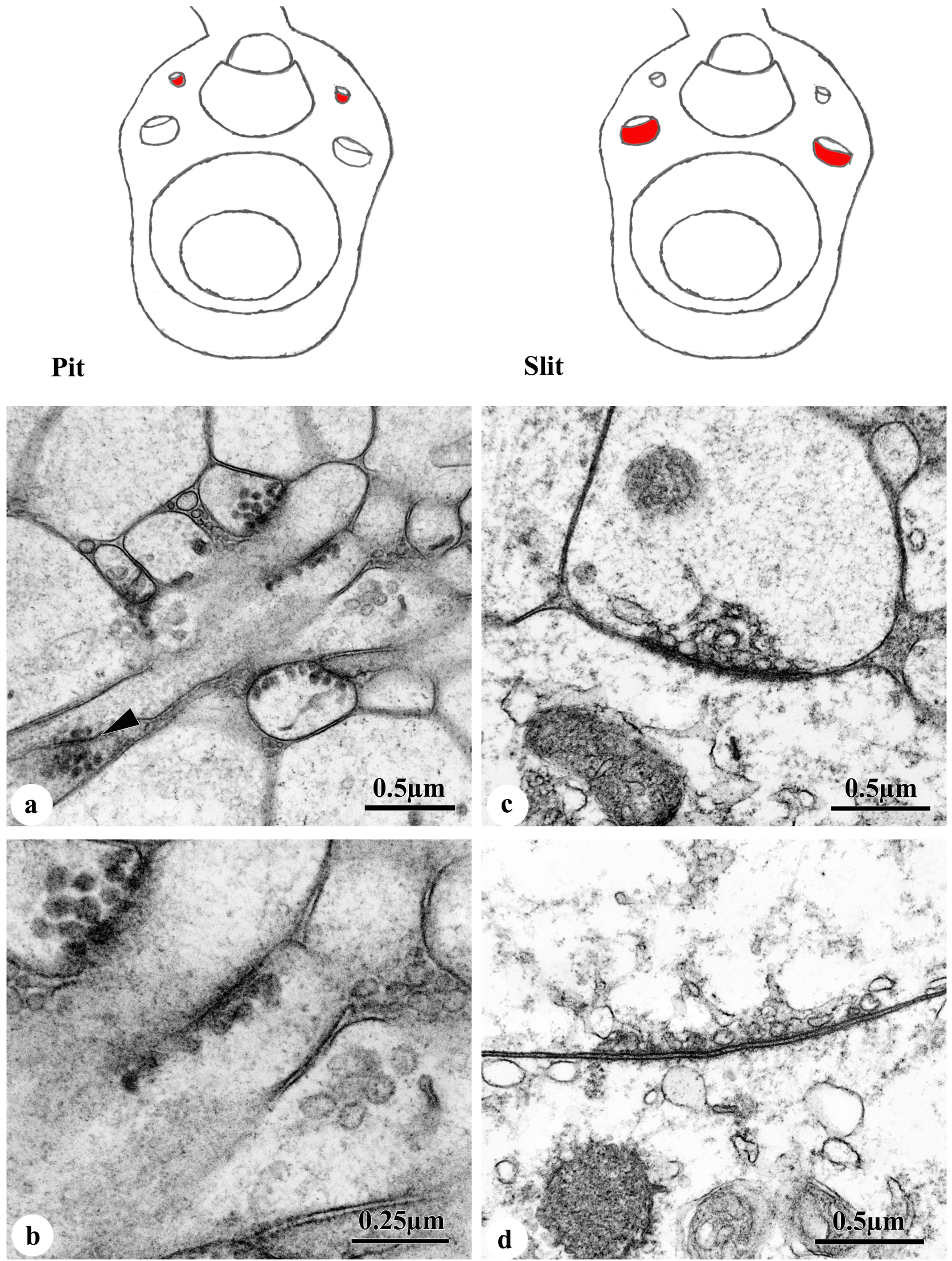
Three different types of synaptic terminals were observed in the pacemaker region (Fig. 15).

Two types of unidirectional synapses were observed, containing stacks of clear or dense core vesicles, about $0.09 \mu \mathrm{m}$ in diameter, located along the synaptic junction. The third type consisted

of bidirectional synapses that were frequently observed between large neurites, which contained relatively clear cytoplasm, and smaller neurites that were filled with microtubules. These synapses contained dense core vesicles, ranging in size from $0.05 \mu \mathrm{m}-0.09 \mu \mathrm{m}$, located on the smaller neurite side of the synaptic junction, and clear vesicles, $0.04 \mu \mathrm{m}-0.08 \mu \mathrm{m}$ in diameter, located on the large neurite side (Fig. 15). Mitochondria were also found in close proximity to theses synapses. A thin layer of electron-dense material was associated with the cytoplasm of both the pre and postsynaptic membranes where the vesicles docked.

\section{DISCUSSION}

The morphology of the different types of synapses found in the rhopalia of Carybdea alata, Chiropsalmus quadrumanus, Carybdea marsupialis, and Tripedalia cystophora was examined and five types of synapses were found, one of which was located only in the upper and lower lensed eyes (Table 5). Statistical analyses showed significant differences in the diameter and densities of invaginated synapses between the upper and lower lensed eyes.

Invaginated synapses have been previously reported in the lower lensed eye of Tamoya bursaria (Yamasu and Toshida, 1976), but without mentioning their presence or absence in the other three types of eyes. Using electron microscopy, invaginated synapses were shown to be present in the upper lensed eye of Carybdea alata, Chiropsalmus quadrumanus, Carybdea marsupialis and Tripedalia cystophora. Yamasu and Toshida (1976) also reported that the 
Figure 15. Pacemaker region: a) Area in which the pacemaker region is located in the rhopalium. Only two of the three types are shown. (b \& c) Type 1- Synapses consist of dense core vesicles located both along the membrane, and throughout the cytoplasm. (d \& e) Type 3Note the distinct difference between the cytoplasm of the larger neurites (ln) and the small neurites (sn). A pattern is seen between vesicle placements. Clear vesicles (arrowheads) were found only on the larger neurite side, whereas dense core vesicles (arrows) were found on the smaller neurites surrounding the large neurite. Insert shows close up of synaptic junctions. Scale bar $=0.25 \mu \mathrm{m}$ 


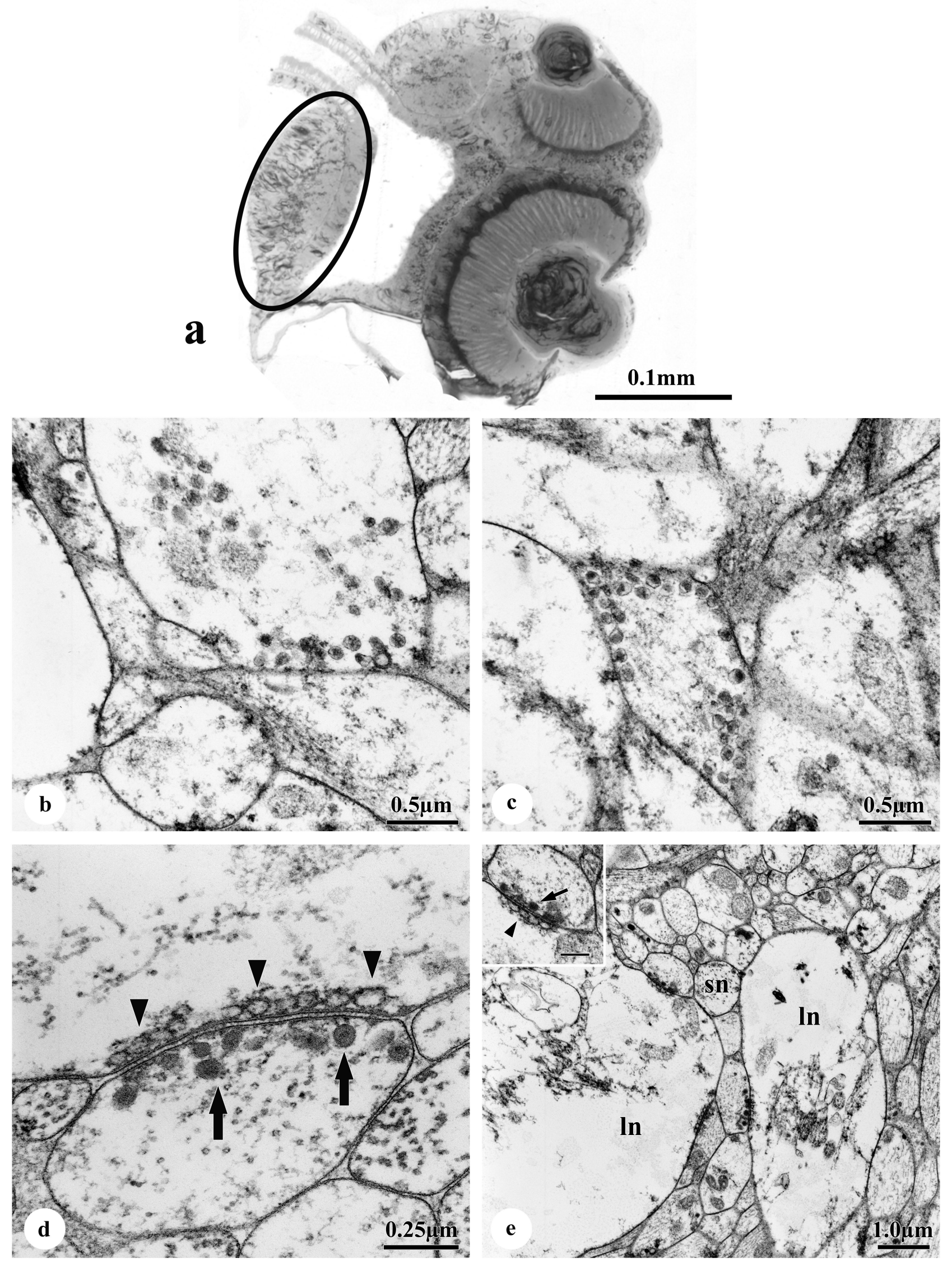


Table 5. Types of synapses found in cubomedusae. Brackets indicate types of synapses reported in other studies: Data from Garm et al., 2007 (G07); Garm et al., 2006 (G06); Skogh et al., 2006 (S); and Yamasu and Yoshida, 1976 (YY).

Species clear dense clear/dense clear invaginated unidirectional unidirectional bidirectional bidirectional synapses

C. alata

Upper lensed eye $\quad+$

Lower lensed eye $\quad+$

Pit $\quad+$

Slit

Pacemaker

$+$

$+$

C. marsupialis

Upper lensed eye

Lower lensed eye

Pit

Slit

Pacemaker

$+$

$+$

$+$

$+$

$+$

$+$

$+$

$+$

C. quadrumanus

Upper lensed eye +

Lower lensed eye

Pit

Slit

Pacemaker

T. cystophora

Upper lensed eye

Lower lensed eye

Pit

Slit

Pacemaker

Stalk

Nerve ring

(S)

(G06)

(G07)

$+$

$+$

$+$

T. bursaria

Lower lensed eye

$+$

$+$

$+$

$+$

$+$

$+$

$+$

$+$

(G06)

(G07) 
synapses invaginated into sensory cells to a depth of $0.25 \mu \mathrm{m}$, which is much shorter than the longest invagination $(0.629 \mu \mathrm{m})$, measured in Carybdea alata.

Invaginated synapses in the lensed eyes of cubomedusae differ from known types of synapses found in cnidarians, therefore they are assumed to be a unique characteristic of cubozoans. Similar invaginations have been observed in other invertebrates, some referred to as spine synapses. In the photoreceptors of Loligo pealei, spine synapses were associated with a narrow 2 to $4 \mathrm{~nm}$ synaptic cleft, which suggests the possibility of electrical junctions (Cohen, 1973). No such narrowing of the synaptic cleft was seen in the invaginated synapses of cubomedusae. In fact, the synaptic cleft width (10-17 nm) indicated that these are indeed, chemical synapses. Similarities are also seen in the type of invagination, with the postsynaptic element invaginating into the presynaptic cells in both Octopus vulgaris and Aplysia californica (Budelmann and Thies, 1977; Bailey and Thompson, 1979). In Aplysia californica, spine synapses were found in the siphon nerve and abdominal ganglion (Bailey and Thompson, 1979), and in the octopus, they have been found in both the statocyst and brain (Budelmann and Thies, 1977).

Bailey and Thompson (1979) suggested that invaginated synapses are more effective at transmitting signals since in Aplysia californica, indented synapses have twice as many vesicles at active zones than do flat synapses. Although vesicle densities were not measured in this study, it could be possible that an increase in surface area, due to the invagination in cubomedusae, could reflect a greater synaptic efficacy than flat cnidarian synapses which typically have few vesicles present (Westfall, 1987).

Similar invaginations of postsynaptic processes are also shown at several specialized synapses, such as the photoreceptor cells in vertebrate retinas (Bullock and Horridge, 1965). These types of synapses are referred to as ribbon synapses. The invaginated synapses in 
cubozoans are similar to the ribbon synapses found in vertebrate eyes, although cubozoans have no synaptic ribbons.

No significant differences in the diameter of the invaginated synapses were observed between Carybdea alata and Carybdea marsupialis. Significant differences however, were observed between the sizes of the invaginated synapses in Chiropsalmus quadrumanus when compared to the other two species, Carybdea alata and Carybdea marsupialis. Chiropsalmus quadrumanus are not in the same family as the other two species, suggesting a difference may exist in synaptic morphology between families.

The presence of synaptic vesicles indicated the use of neurotransmitters as the mode of communication between processes. Positive labeling for RFamide, glutamate, serotonin, and GABA, has been reported in the areas in which invaginated synapses are found (Martin, 2002). These same neurotransmitters are known to function in the photobiology of vertebrates and invertebrates (Lam et al., 1982). Their presence, however, has not been verified in the synaptic vesicles of invaginated synapses.

Significant differences exist in the densities between the lower and upper lensed eyes in Carybdea alata and Chiropsalmus quadrumanus. Although it is not clear why these differences in synaptic density occur, one possibility is that a correlation may exist between invaginated synapses and a particular type of photoreceptor. Visual behavior experiments (Garm et al., 2007b) have suggested that the upper and lower lensed eyes have different functional roles in visual detection. Presently, it is unknown what role the upper lensed eye plays in visual behavior, however, Garm et al. (2007b) suggested that the lower lensed eye is used more than the upper lensed eye in mediating obstacle avoidance responses. Also, morphological differences in photoreceptor populations in the upper and lower lensed eyes have been demonstrated in 
Carybdea marsupialis (Martin, 2004). Further analysis, however, is needed to determine if different populations of photoreceptors in Carybdea alata, Chiropsalmus quadrumanus, and Tripedalia cystophora are present. Such findings may explain the differences in the densities observed.

The identification of invaginated synapses between putative photoreceptor cells in both Carybdea alata and Carybdea marsupialis suggest some type of direct communication between photoreceptor cells. The obstacle avoidance experiments on Tripedalia cystophora and Chiropsella bronzie (Garm et al., 2007b) suggest some degree of spatial resolution in the lensed eye, which Nilsson et al. (2005) reported may be the case in the lower lensed eye of Tripedalia cystophora. It is possible that some form of lateral inhibition could be taking place, which would increase the spatial resolution in both the upper and lower lensed eyes. The density measurements indicated a large number of invaginated synapses found near the photoreceptor cells, which suggests that the first part of visual processing may be taking place in this zone.

Specialization in the basal region of many types of receptor cells allows them to form a presynaptic zone (comprised of synaptic vesicles and membrane thickenings), which is located at the region opposite the second order neurite (Gray, 1971). In cubozoans, the basal end of the photoreceptor cells tapers and projects into the neural plexus of the retina (Yamasu and Toshida, 1976; Martin, 2004). This corresponds to zone 2 in this study. In Carybdea alata, Carybdea marsupialis, and Chiropsalmus quadrumanus, the highest densities of invaginated synapses are located at, or near, the base of the photoreceptor cells. For those cell processes that could not be identified in zone 2, it is possible some may be the branching process of the photoreceptor cells. Martin (2004) reported photoreceptor cells ranging in length from 50-60 $\mu \mathrm{m}$, indicating that the majority of the branching process of the photoreceptor cells would be located in zone 2 . 
A unique correlation was observed between the presence of mitochondria and the location of invaginated synapses. Mitochondria are commonly found in zones 1 \& 2 where the majority of invaginated synapses were located in Carybdea alata, Chiropsalmus quadrumanus, and Carybdea marsupialis. The sparseness of mitochondria in zone 3, along with the lack of synapses, suggests that this zone is an area of impulse conduction.

It is possible that invaginated synapses were not observed in other areas due to the way in which the tissue was sectioned. Multiple sections ( $~ 90 \mathrm{~nm} / 5$ sections per grid and at least 3 grids per structure) were examined to locate these structures and to determine their location within the eye. Also, with the synapses invaginating into the presynaptic photoreceptors to a depth of 0.62 $\mu \mathrm{m}$, it is unlikely these synapses were missed in the sections taken. This also suggests that invaginated synapses are absent in the pit and slit ocelli.

The types of chemical synapses observed in the pit and slit ocelli, and in the pacemaker region, are commonly found in other cnidarians (see Westfall, 1987). The unidirectional synapses observed in the pit, slit, and lensed eyes are similar to the synapses found in the ocelli region of the hydrozoan, Polyorchis penicillatus. In Polyorchis, unidirectional synapses are located on either the receptor cell side, or second order neuron cell side, and have granular synaptic vesicles 60- $80 \mathrm{~nm}$ in diameter (Singla and Weber, 1982a). Also, the dense core vesicles found in the cup ocelli of the hydrozoan, Sarsia tubulosa (Singla and Weber, 1982b) are very similar to those found in the eyes of cubozoans, although the synaptic vesicles were much larger $(65-180 \mathrm{~nm})$ in Sarsia.

The role of the unidirectional and bidirectional synapses in the pit, slit, lensed eyes, and pacemaker region is unclear. In other cnidarians, unidirectional and bidirectional synapses contact a single postsynaptic element (Westfall, 1987). In this study, however, in addition to the 
observation of single contacts, multiple contacts were also observed in the pit and lensed eyes. Unidirectional and bidirectional synapses have been seen in the stalk and nerve ring in Tripedalia cystophora (Garm et al., 2006, 2007a) as well as on photoreceptors axon terminals in hydrozoans (Singla and Weber, 1982a, 1982b).

Carybdea alata are unique in having bidirectional chemical synapses in which clear vesicles are found only on one side of the synaptic junction, and dense core vesicles are found on the other side. The presence of two different types of synaptic vesicles within these synapses suggests a form of potential polarization in a bidirectional synapse.

The present study supports the hypothesis that invaginated synapses are only located in the upper and lower lensed eyes of these four species of cubomedusae. Similar observations in all four species suggest that invaginated synapses can indeed be used as markers to help sort out the rhopalial circuitry. The synaptic morphology of the rhopalia suggests that the first level of visual processing occurs in the retina in Carybdea alata, Chiropsalmus quadrumanus, Carybdea marsupialis, and Tripedalia cystophora. The density results from this study support the hypothesis that invaginated synapses are in close association to photoreceptor cells. This study is the first to show that cubomedusae photoreceptor cells send out postsynaptic processes into surrounding cells, and suggests that invaginated synapses may be correlated to a particular type of photoreceptor cell.

This study provided a descriptive account of the synaptic morphology of the cubomedusan rhopalium and represents a first step is sorting out the synaptic organization of cubomedusan eyes. It also produced a structural framework in which synaptic organization in the rhopalia can be better understood. An important feature of any visual system is the ability to integrate visual information obtained from the surrounding environment. In most bilateral organisms, 
information is received by sensory systems (eyes), which make connections to a cephalized nervous system. The central nervous system then sorts the information and passes the information on to the appropriate effectors. In cubomedusae, the CNS is radially arranged and considered to be relatively simple. The elaborate visual behaviors of cubomedusae, however, suggest the presence of a central nervous system that is not as poorly developed as previously thought (Garm et al., 2006). Ultrastructural, immunohistochemical, and electrophysiological work have shown the rhopalia to have a complex nervous system with distinct, directional neural pathways (Garm et al., 2006; Garm et al., 2007a; Parkefelt et al., 2005; Skogh et al., 2006; Satterlie, 1979; Satterlie and Spencer, 1979; Satterlie and Nolen, 2001), suggesting the rhopalia may be both structurally and physiologically similar to ganglia of bilateral invertebrates. 


\section{LITERATURE CITED}

Bailey, C. H., and E. B. Thompson. 1979. Indented synapses in Aplysia. Brain Res. 173: 13-20.

Barnes, J. H. 1961. Studies on three venomous cubomedusae. Pp. 307-332 in The Cnidaria and Their Evolution, W.J. Rees, eds. Zoological Society of London.

Berger, E. W. 1898. The histological structure of the eyes of cubomedusae. J. Comp. Neurol. 8: 223-230.

Berger, E. W. 1900. Physiology and histology of the cubomedusae. Mem. Biol. Lab. Johns Hopkins 4(4): 1-84.

Budelmann, Bernd-Ulrich, and G. Thies. 1977. Secondary sensory cells in the gravity receptor system of the statocyst of Octopus vulgaris. Cell Tiss. Res. 182: 93-98.

Burr, A. H. 1984. Evolution of eyes and photoreceptor organelles in the lower phyla. Pp. 131178 in Photoreceptors and vision in invertebrates, M. A. Ali, eds. Plenum Press, New York.

Coates, M. M. 2003. Visual ecology and functional morphology of Cubozoa (Cnidaria). Integr. Comp. Biol. 43: 542-548.

Coates, M. M. 2004. Vision in a cubozoan jellyfish, Tripedalia cystophora. Ph.D. dissertation, Hopkins Marine Station, Stanford University, Stanford.

Coates, M. M., and J. C. Theobald. 2004. Optimal visual parameters for a cubozoan jellyfish in the mangrove environment. In Society for Integrative and Comparative Biology Annual Meeting New Orleans. 316 (Abstract).

Conant, F.S.1898. Cubomedusae. Mem. Biol. Lab. Johns Hopkins 4(1): 1-61.

Cohen, A. I. 1973. An ultrastructural analysis of the photoreceptors of the squid and their synaptic connections III. Photoreceptor termination in the optic lobes. J. Comp. Neur. 147: 399426.

Conant, F. S. 1898. The cubomedusae. Mem. Biol. Lab. Johns Hopkins. 4(1): 1-61.

Garm, A., P. Ekstrom, M. Boudes, and D. E. Nilsson. 2006. Rhopalia are integrated parts of the central nervous system in box jellyfish. Cell Tiss. Res. 2: 333-343.

Garm, A., M. M. Coates, R. Gad, J. Seymour, and D. E. Nilsson. 2007a. The lens eyes of the box jellyfish, Tripedalia cystophora and Chiropsalmus sp. are slow and color-blind. J. Comp. Physiol A. 
Garm, A., M. O’Connor, L. Parkefelt, and D. E. Nilsson. 2007b. Visually guided obstacle avoidance in the box jellyfish, Tripedalia cystophora and Chiropsella bronzie. J. Exp. Biol. 210: 3616-3623.

Gray, E.G. 1971. The fine structural characterization of different types of synapse. Prog Brain Res. 34: 149-160.

Hamner, W. M. 1994. Australia's box jellyfish: a killer down under. Natl. Geogr. Mag. 186: 116-130.

Hamner, W. M., M. S. Jones, and P. P. Hamner. 1995. Swimming, feeding, circulation and vision in the Australian box jellyfish, Chironex fleckeri (Cnidaria, Cubozoa). Mar. Freshw. Res. 46: 985-990.

Lam, D. M., K. Frederick, J. M. Hollyfield, J. G. P. Sarthy, and R. E. Marc. 1982. Identification of neurotransmitter candidates in invertebrate and vertebrate photoreceptors. Pp. 65-80 in Visual cells in evolution. J.A. Westfall, eds. Raven Press, New York.

Land, M. F., and D. E. Nilsson. 2002. Animal Eyes. Oxford Univ. Press, Oxford.

Larson, R. J. 1976. Cubomedusae: feeding, functional morphology, behavior, and phylogenetic position. Pp. 237-245 in Coelenterate Ecology and Behavior, G. O. Mackie, eds. Plenum Press, New York.

Martin, V. J. 2000. Box jellyfish may be able to see. Regional perspectives in marine biology. McGraw-Hill Co, New York.

Martin, V. J. 2002. Photoreceptors of cnidarians. Can. J. Zool. 80: 1703-1722.

Martin, V. J. 2004. Photoreceptors of cubozoan jellyfish. Hydrobiologia. 530/531: 135-144.

Matsumoto, G. I. 1995. Observations on the anatomy and behavior of the cubozoan, Carybdea rastonni Haacke. Mar. Freshw. Behav. Physiol. 26: 139-148.

Millonig, G. 1961. Advantages of a phosphate buffer for $\mathrm{OsO}_{4}$ solution in fixation. J. Appl. Phys. 32: 1637.

Nilsson, D. E. 1989. Vision optics and evolution: Nature's engineering has produced astonishing diversity in eye design. Bioscience. 39: 298-307.

Nilsson, D. E., L. Gislen, M. M. Coates, C. Skogh, and A. Garm. 2005. Advanced optics in a jellyfish eye. Nature 435: 201-205.

Parkefelt, L., C. Skogh, D. E. Nilsson, and P. Ekstrom. 2005. Bilateral symmetric organization of neural elements in the visual system of coelenterate, Tripedalia cystophora. J. Comp. Neur. 492: 251-262. 
Piatigorsky, J., J. Horwitz, T. Kuwabara, and C. E. Cutress. 1989. The cellular eye lens and crystallins of cubomedusan jellyfish. J. Comp. Physiol. 164: 577-587.

Reynolds, E. S. 1963. The use of lead citrate at high $\mathrm{pH}$ as an electron-opaque stain in electron microscopy. J. Cell Biol. 17: 208-212.

Satterlie, R. A. 1979. Central control of swimming in the cubomedusan jellyfish, Carybdea rastonni. J. Comp. Physiol. 133: 357-367.

Satterlie, R. A., and Nolen, T. G., 2001. Why do cubomedusae have only four swim pacemakers? J. Exp. Biol. 204: 1413-1419.

Satterlie, R. A., and A. N. Spencer. 1979. Swimming control in a cubomedusan jellyfish. Nature 231: 141-142.

Singla, C. L., and C. Weber. 1982a. Fine structure studies of the ocelli of Polyorchis penicillatus (Hydrozoa, Anthomedusae) and their connection with the nerve ring. Zoomorphology. 99: 117-129.

Singla, C. L., and C. Weber. 1982b. Fine structure of the ocellus of Sarsia tubulosa (Hydrozoa, Anthomedusae). Zoomorphology. 100: 11-22.

Skogh, C., D. Garm, E. Nilsson, and P. Ekstrom. 2006. Bilaterally symmetrical rhopalial nervous system of the box jellyfish, Tripedalia cystophora. J. Morph. 267: 1391-1405.

Spurr, R. A. 1969. A low- viscosity epoxy resin embedding medium for electron microscopy. $J$. Ultrastruct Res. 26: 31-43.

Stewart, S. E. 1996. Field behavior of Tripedalia cystophora (class Cubozoa). Mar.Freshw. Behav. Physiol. 27: 175-188.

Werner, B. 1973. New investigation on sytematics and evolution of the class, Scyphozoa and the phylum, Cnidaria. In Recent trends in Research in Coelenterate Biology. 35-59.

Westfall, J. A. 1987. Ultrastucture of invertebrate synapses. Pp. 3-28 in Nervous systems in invertebrates, M. A. Ali, eds. Plenum Press, New York.

Yamasu, T., and M. Yoshida. 1976. Fine structure of complex ocelli of a cubomedusan, Tamoya biirsaria Haeckel. Cell Tiss. Res. 170: 325-329. 